Draft Version March 8, 2022

Preprint typeset using $\mathrm{AT}_{\mathrm{E}} \mathrm{X}$ style emulateapj v. 04/21/05

\title{
COMPARING THE HOST GALAXIES OF TYPE IA, TYPE II AND TYPE IBC SUPERNOVAE
}

\author{
X. Shao ${ }^{1,2,3,5}$, Y. C. Liang ${ }^{1}$, M. Dennefeld ${ }^{4}$, X. Y. Chen ${ }^{1,3}$, G. H. Zhong ${ }^{1,3}$, F. Hammer ${ }^{5}$, L. C. Deng ${ }^{1}$, H. Flores ${ }^{5}$, \\ B. ZHANG ${ }^{1,2}$, W. B. Shi ${ }^{1,6}$ AND L. Zhou ${ }^{1,3,6}$ \\ (Dated: Received / Accepted) \\ Draft version March 8, 2022
}

\begin{abstract}
We compare the host galaxies of 902 supernovae, including SNe Ia, SNe II and SNe Ibc, which are selected by cross-matching the Asiago Supernova Catalog with the SDSS Data Release 7 . We further selected 213 galaxies by requiring the light fraction of spectral observations $>15 \%$, which could represent well the global properties of the galaxies. Among them, 135 galaxies appear on the Baldwin-Phillips-Terlevich diagram, which allows us to compare the hosts in terms of star-forming, AGNs (including composites, LINERs and Seyfert 2s) and "Absorp" (their related emission-lines are weak or non-existence) galaxies. The diagrams related to parameters $\mathrm{D}_{n}(4000), \mathrm{H} \delta_{A}$, stellar masses, SFRs and specific SFRs for the SNe hosts show that almost all SNe II and most of SNe Ibc occur in SF galaxies, which have a wide range of stellar mass and low $\mathrm{D}_{n}(4000)$. The SNe Ia hosts as SF galaxies follow similar trends. A significant fraction of SNe Ia occurs in AGNs and Absorp galaxies, which are massive and have high $\mathrm{D}_{n}(4000)$. The stellar population analysis from spectral synthesis fitting shows that the hosts of SNe II have a younger stellar population than hosts of SNe Ia. These results are compared with those of the 689 comparison galaxies where the SDSS fiber captures less than $15 \%$ of the total light. These comparison galaxies appear biased towards higher $12+\log (\mathrm{O} / \mathrm{H})$ $(\sim 0.1 \mathrm{dex})$ at a given stellar mass. Therefore, we believe the aperture effect should be kept in mind when the properties of the hosts for different types of SNe are discussed.

Subject headings: Galaxies: abundances - Galaxies: evolution-Galaxies: formation - Galaxies: spiral Galaxies: starburst - Galaxies: star formation
\end{abstract}

\section{INTRODUCTION}

Supernovae (SNe) are classified into various types (II, $\mathrm{Ib}$, Ic and Ia) according to the presence or absence of various features in their spectra (Filippenko (1997) and reference therein). The presence or absence of hydrogen distinguishes type II from type I SNe, respectively. Among the type I's, the presence of Si lines characterizes types Ia, while the presence of He lines distinguishes types Ib from types Ic's (Hamuv et al. (2002); Turatto (2003), and references therein).

$\mathrm{SNe}$ Ia are observed in all types of galaxies (ellipticals, irregulars, spirals), suggesting that they are somehow connected to the evolution of less-massive stars (e.g., Oemler \& Tinslev (1979); van den Bergh (1990); della Valle \& Livio (1994); Cappellaro et al. (1999)). It is widely accepted that the progenitors of Ia $\mathrm{SNe}$ are carbon-oxygen white dwarfs (CO WD's), which have accreted material up to the Chandrasekhar limit (Chandrasekhar 1931) from non-degenerate companion stars) in a single-degenerate (SD) model (Whelan \& Iben 1973; Nomoto 1982), or come from a double-degenerate

\footnotetext{
Electronic address: xshao@bao.ac.cn,ycliang@bao.ac.cn

1 Key Laboratory of Optical Astronomy, National Astronomical Observatories, Chinese Academy of Sciences, 20A Datun Road, Chaoyang District, Beijing, 100012, China

2 Department of Physics, Hebei Normal University, Shijiazhuang 050016, China

${ }^{3}$ Graduate University of the Chinese Academy of Sciences, 19A Yuquan Road, Shijingshan District, 100049, Beijing, China

${ }^{4}$ Institut d'Astrophysique de Paris, CNRS, and Universite P. et M. Curie, 98bis Bd Arago, F-75014 Paris, France

${ }^{5}$ GEPI,Observatoire de Paris-Meudon, 92195 Meudon, France

${ }^{6}$ Shandong Provincial Key Laboratory of Optical Astronomy and Solar-Terrestrial Environment, School of Space Science and Physics, Shandong University at Weihai, Weihai 264209, China
}

(DD) model, which involves the merger of two CO WDs (Iben \& Tutukov 1984; Webbink 1984).

However, type II, Ib and Ic SNe are only found in star-forming galaxies, indicating that they are the product of evolution in massive stars from the gravitational collapse of their Fe cores. Thus, type II and type Ibc (which include types Ib, Ic and Ib/c) SNe are also called core collapse $\mathrm{SNe}$ (CC-SNe). CC-SNe are thought to arise from stars with initial masses $>8 M_{\odot}$ : this value results from the agreement between direct detections of progenitors (Smartt 2009), and the maximum observed mass for white dwarfs (WD's) (Williams et al. 2009; : Anderson \& James 2009).

Many studies have tried to understand how various types of supernovae behave, including mass of progenitors, effect of environments and the relations between the host properties and the SNe themselves etc., but these were generally only based on small samples (Hamuy et al. (1995, 1996, 2000); Gallagher et al. (2005, 2008); Neill et al. (2009) for SNe Ia; Anderson \& James (2009); Habergham et al. (2010) for CCSN).

The studied sample has been greatly extended in recent years, especially benefitting from the successful projects associated with SDSS, both for galaxies (Strauss et al. 2002; Kauffmann et al. 2003a,b; Brinchmann et al.|2004; Tremonti et al. 2004) and supernovae (e.g. the SDSSII Supernova Survey: Frieman et al. (2008); Zheng et al. (2008); Lampeitl et al. (2010); D'Andrea et al. (2010); Cooper et al. (2009)). The enlarged sample makes it possible to carefully compare the properties of host galaxies of supernova explosions. Some comparison studies have been made that examine the differences between galaxies that host SNe Ia and CC-SNe, separately. Han et al. 
(2010) investigated the color, luminosity and environments of SNe Ia host galaxies in Stripe 82 of the SDSSII Supernova Survey centered on the celestial equator. Kelly \& Kirshner (2012) examined the host galaxies of core-collapse supernovae where they separately inspected colors at the sites of the explosions, the chemical abundances and specific star formation rates for hosts of SNe II, SNe IIn, SNe IIb, SNe Ib and SNe Ic.

However, there are few works that combine SNe Ia and CC-SNe together to compare their hosts. This is interesting and useful for understanding the environments where SNe explode, especially because some SNe Ia hosts are also star-forming galaxies, like those for SNe II and $\mathrm{SNe}$ Ibc. Some researches have even tried to do so, but they have only focused on some limited aspects of the properties, for example, Prieto et al. (2008) mostly discussed the metallicities of galaxies, Hakobyan et al. (2012) mostly reported the creation of their database from SDSS-DR8 and presented some measurements from images. Therefore, many more comparisons are needed for studying SNe host galaxies, such as stellar populations, stellar mass and star formation rates (SFRs) and so on. These properties are very important for understanding the characteristics of supernova hosts.

In this work, we take into account SNe Ia, SNe II and $\mathrm{SNe}$ Ibc (the later two as CC-SNe) together to compare the properties of their hosts. In particular, we will use a stricter selection criterion to select the objects, for which the 3 arcsec fiber spectra of SDSS can represent the global properties of the galaxies better. We believe it is important to show the global properties of supernova host galaxies since it is often difficult to acquire the local properties at the sites of an SN explosion.

Our idea can be summarized as follows: 1) Comparing the host galaxies of all kinds of supernovae, including both Type Ia and CC-SNe (SNe II and Ibc). 2) We will compare them in terms of parameters that describe many properties, including stellar masses, star formation rates, specific star formation rates, $\mathrm{D}_{n}(4000), \mathrm{H} \delta_{A}$ and gas-phase oxygen abundances. 3) We also run spectral synthesis analysis for the optical spectra and obtain the light weighted average ages for the host galaxies. 4) The hosts can be carefully checked following the classification from their high quality emission-line ratios, which can diagnose the hosts on the BPT diagram (Baldwin et al. 1981). Then star forming (SF), AGN and Absorption line galaxies acting as hosts can be compared in terms of these property relations. 5) Since the sample is large, it is possible for us to manage a good sub-sample that can represent the global properties of the host galaxies, for which the 3 arcsec SDSS fiber observations can cover $>15 \%$ light. This minimizes the cases that the fiber only record a small part of the global light of the hosts. 6) Then we can also carefully discuss the aperture effect of the SDSS fiber observations by comparing the two sub-samples (the ones that have light fraction $>15 \%$ and the others that have light fraction $\leq 15 \%$ ); this approach gives clearer results for stellar mass-metallicity relations. 7) The properties of SNe hosts mentioned above will also be compared with the main sample of galaxies from SDSS-DR7.

This paper is organized as follows. We describe the sample selection in Sect. 2, which demonstrates how we select the 213 sample galaxies with light fraction higher than $15 \%$ from the SDSS fiber observations, and the selection for the comparative sample galaxies are also mentioned. The parameters describing properties and their relations are shown in Sect. 3 for these hosts. Results of the stellar population analysis are presented in Sect. 4. Discussions are presented in Sect. 5, where we show the results of comparisons with the 689 comparative sample galaxies, the stellar mass-metallicity relation and the aperture effect/bias. Conclusions are given in Sect. 6 . Throughout the paper, a cosmological model with $\mathrm{H}_{0}=$ $70 \mathrm{~km} \mathrm{~s}^{-1} \mathrm{Mpc}^{-1}, \Omega_{M}=0.3$ and $\Omega_{\Lambda}=0.7$ is adopted.

\section{SAMPLE SELECTION}

We select the $\mathrm{SNe}$ and their host galaxies by crossmatching the Asiago Supernova Catalog (ASC) with the SDSS DR7 main galaxy sample (MGS), only retaining spectral observations of the SNe host galaxies with good quality.

\subsection{The Asiago Supernova Catalog (ASC)}

The ASC was first published in 1984 by Barbon et al., who assembled all the pertinent information on the 568 Supernovae (SNe) discovered from 1885 up to 1983, as well as some parameters associated with their host galaxies. The catalog was subsequently updated for newly discovered SNe. Barbon et al. (1989), Barbon et al. (1999) and their group made the following updates. Up to the end of 2013, the Asiago supernova catalog includes 6312 SNe up to SNe 2013hx (http://graspa.oapd.inaf.it).

In this work, we adopted the RA and DEC of the SNe host galaxies in the ASC table, to enable cross-matching with the SDSS-DR7 MGS galaxies.

\subsection{The SDSS main galaxy sample catalog (MGS)}

The SDSS is the most ambitious astronomical survey ever undertaken in imaging and spectroscopy (York et al. 2000; Stoughton et al. 2002; Abazajian et al. 2003, 2004). The imaging data are done in drift scan mode and are $95 \%$ complete for the surveyed area for point sources at 22.0,22.2, 22.2, 21.3 and 20.5 in five bands $(u, g, r, i$ and $z$ ) respectively. The spectra are flux- and wavelength-calibrated from 3800 to $9200 \AA$ at $R \approx 1800$.

The sample used in this work is selected from the SDSS-DR7 MGS (Strauss et al. 2002), which comprises galaxies with $r$-band Petrosian magnitude $14.5<r \leq 17.77$ (corrected for foreground Galactic extinction using the reddening maps of Schlegel et al. (1998) ) and $r$-band Petrosian half-light surface brightness $\mu_{50} \leq 24.5 \mathrm{mag} \operatorname{arcsec}^{-2}$.

The parameters of the galaxies in SDSS-DR7 have been derived and published by the MPA/JHU group ${ }^{7}$. We adopt their emission-line measurements and some properties of the galaxies, such as $\mathrm{D}_{n}(4000), \mathrm{H} \delta_{A}$, stellar masses, star formation rates, metallicities etc. for the present work.

\subsection{Cross-correlations of $A S C$ and $S D S S-M G S$}

To select the working sample of SNe host galaxies, we cross-correlated the coordinates of SNe host galaxies from ASC and the coordinates of spectral observations

\footnotetext{
7 http://www.mpa-garching.mpg.de/SDSS/
} 
of the SDSS galaxies in their MGS with the following criteria.

1. Selecting those with well-defined SNe types - We firstly select the supernovae and their host galaxies from the updated ASC (up to SN 2013Y, 6105 samples in total). Then only those having well-defined types of SNe, e.g., type Ia, II and Ib/c, are further selected. This is 4,934 samples. It is worth noting here that the ASC contains events since 1885, and that a precise sub-classification, particularly of type I's, did not exist at the beginning of research about $\mathrm{SNe}$.

2. First cross-matching with $30^{\prime \prime}$ radius - In the $\mathrm{ASC}$, we notice that the coordinates of the $\mathrm{SNe}$ host galaxies are not accurate enough in both RA (h:m:s) and DEC (d:m:s) since both terms of "s" only down to integers without decimals. This could cause obvious discrepancy when matching the two catalogs, which may be up to 15 arcseconds for RA (if the discrepancy up to $\pm 1 \mathrm{~s}$ ) and a bit less for DEC.

To minimize this problem, we adopt two steps for matching coordinates to select the final working sample. In the first, we adopt 30 arcsec as the matching radius to cross-correlate the coordinates of the 4,934 $\mathrm{SNe}$ host galaxies in ASC with the SDSS DR7 MGS (698,260 entries). This radius is large enough for considering the low precision of both RA and DEC. This value is half the radius for matching used by Prieto et al. (2008). With this 1,105 host galaxies are selected, by removing the duplicated and multiple spectral observations, 1,041 host galaxies are retained.

3. Coordinate corrections - In order to improve the reliability of cross-matching between ASC and SDSS, we try to improve the accuracy of the coordinates of the SNe hosts in ASC. To do so, we retrieve the International Celestial Reference System (ICRS) coordinates (epoch in J2000) from SIMBAD (http://simbad.u-strasbg.fr) for the hosts by searching them by designation. The ICRS coordinates have two or three decimals for "s" terms in RA and DEC, which could improve the accuracy of matching between ASC and SDSS catalogs for SNe host galaxies. 687 (among 1,041) host galaxies are obtained their ICRS coordinates from SIMBAD, but the other 354 ones could not be found there, so we had to keep their original coordinates from ASC.

To show the necessity and significance of the improvement in such coordinate precision for the $\mathrm{SNe}$ host galaxies, in Fig. 1 we plot the difference between the coordinates of the 687 hosts from the ASC and those of the ICRS ones, which shows the low precision of the coordinates in ASC. Now we have a new table for 1,041 SNe hosts, and 687 of them have updated RA and DEC from ICRS.

4. Second cross-matching with $15^{\prime \prime}$ radius - We prefer a smaller radius for matching the two catalogs since it will help to avoid the mis-matching cases. Therefore, we adopt 15 arcsec as the matching radius to redo the cross-correlation for the 1,041 SNe host galaxies from ASC and SDSS-DR7 MGS. Among them, 687 objects had revised RA-DEC as ICRS coordinates from SIMBAD as mentioned above. After this step, 980 objects were obtained (where 672 are from the 687 sample and 308 are from the 354 sample).

Hopefully, the 15 arcsec matching radius here can help to select as many interesting objects as possible, and to avoid the mistaken cases of crossmatching.

\subsection{Light fraction criterion for global properties from fiber spectral observations}

The aperture of the fiber is 3 arcsec in the SDSS observations. In this study, we mostly focus on the global properties of the host galaxies from SDSS spectra, thus we further select the sample galaxies which have more than $15 \%$ of their light covered by the fiber observations. This light fraction criterion $(>15 \%)$ could help us to select the cases in which the 3 arcsec fiber observations of SDSS cover most of the light of the whole galaxy and thus retrieve the global properties of the SNe host galaxies.

To judge this, one simple and accurate way is to compare the "fiber" and "petrosian" magnitudes of the SDSS galaxies. The fiber mag is a measurement of the light going down the fiber and the Petrosian mag is a good estimate of the total magnitude. Thus, we adopt the formula below to estimate how much light was covered by the fiber observations in r-band (Liang et al. 2010):

$$
\text { light_fraction }=10^{\left(-0.4 *\left(\text { fiber_mag-petro_mag }_{r}\right)\right.} \text {. }
$$

Fig. 2 shows the relations between the calculated light fractions and the Petrosian radius in r-band for the selected $980 \mathrm{SNe}$ hosts.

From Fig. 2 we can see that for a large part of the SNe hosts, the 3 arcsec fiber of SDSS cannot cover more than $10 \%$ of the light from the whole galaxy. Here a bit stricter criterion is used to select our sample galaxies, which have light fraction larger than 0.15 in the spectral observations. To choose 0.15 of light fraction here, we expect not only a reasonable sample of galaxies are selected for studies, of which the SDSS spectra are representative of the whole galaxy, but also we won't lose too many objects with large size. Although we lose a large fraction of the initial sample by performing such a light fraction cut, we believe it is necessary to guarantee that the spectral observations are able to represent the global light of the galaxies, rather than a small region inside the galaxy. We will discuss the effects of lower light fraction with the comparative sample in Sect. 5, where the host galaxies with light fraction lower than 0.15 will be taken for comparison and then the aperture effect will be shown clearly.

The horizonal line on Fig. 2 shows this light fraction cut. Above the 0.15 line, there are 243 objects. It is clear that this selection biases against close, large galaxy hosts. These 243 galaxies are our main working sample in this work. The remaining 737 galaxies (from the total of 980 objects) having lower light fractions will be taken as comparison and are specially discussed in Sect. 5.1.

Our sample galaxies are plot in the Petrosian radius vs. redshift relation in Fig. 3. We see that our main 
working sample galaxies, denoted by the triangles, have small Petrosian radius.

\subsection{Further criteria}

We do further careful checks for the selected sample galaxies.

1. Image checking by eye - In order to guarantee the correction for host-identification between supernovae and galaxies with SDSS observation, we check the 243 samples case by case and remove 14 cases which have misidentification. For example, the $\mathrm{SNe}$ explosion locates in a faint galaxy, which was not taken spectrum by SDSS observation. Instead the SDSS takes spectrum on a bright galaxies quite close to it. After this step, 229 hosts are left.

2. Spectral observations and quality control - We downloaded the SDSS 1D spectra of these $229 \mathrm{SNe}$ host galaxies. Some of them have to be removed since interruption appear in their spectral energy distribution (SED). After this cleaning, we have 213 SNe host galaxies left, whose S/N (median value per pixel of the whole spectrum provided in the MPA/JHU catalog) are larger than 5 .

In Table 1, we list 6 examples of these 213 objects: the table includes coordinates (RA and DEC of SNe hosts from ASC, RA and DEC from SDSS), PID-MJD-FID numbers of the SDSS spectral observations, Petrion radius in r-band, light fraction, redshift, and the types and names of the supernovae. The table with the entire 213 galaxies will be available in electronic form.

3. Three sub-samples associated with emission-line ratios - Since we are working on the properties of host galaxies of supernovae, it will be interesting to check their emission or absorption lines. In the total sample of 213 SNe host galaxies, 135 have good quality observations in all four emission lines [NII] $6583, \mathrm{H} \alpha$, [OIII] 5007 and $\mathrm{H} \beta$ with an $\mathrm{S} / \mathrm{N}$ better than $3 \sigma$. They can be plotted on the BPT diagram (Baldwin et al. 1981) as shown in Fig. 4. The remaining 78 objects have a lower $\mathrm{S} / \mathrm{N}$ in these four emission lines, or only display some of them or none at all. Then the sample galaxies can be divided into three sub-samples:

1) 82 star-forming (SF) galaxies identified by their emission-line ratios in $\mathrm{BPT}$;

2) 53 AGNs (including composites, LINERs and Seyfert 2s);

3) 78 absorption-line and weak emission-line galaxies (named as "Absorp" simply) which cannot appear on the BPT diagram due to the absence of some or all of the four emission lines mentioned above.

In Sect. 3 we will plot the relations of some parameters associated with properties of the hosts of different types of supernovae, by marking their hosts as SF, AGN and Absorp.

Table 2 shows the numbers of the hosts of the different types of SNe according to the galaxies appearing on the

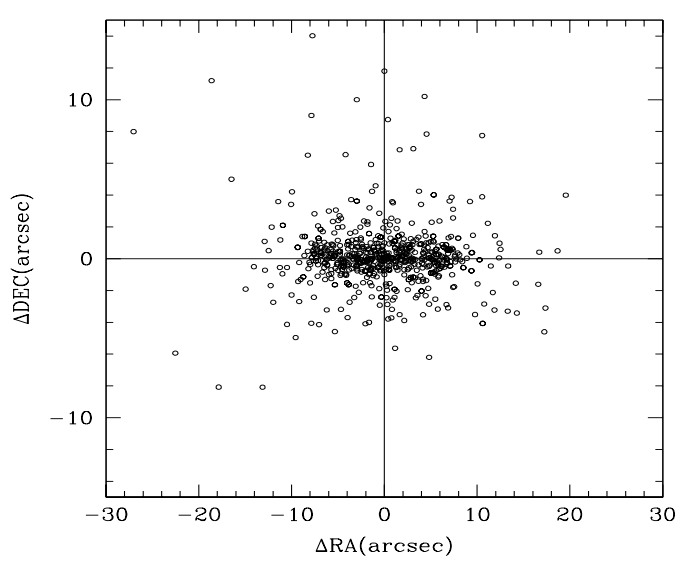

Fig. 1.- The discrepancy between the original coordinates of the 687 SNe host galaxies from the ASC and those from the ICRS from SIMBAD which have the ICRS coordinates.

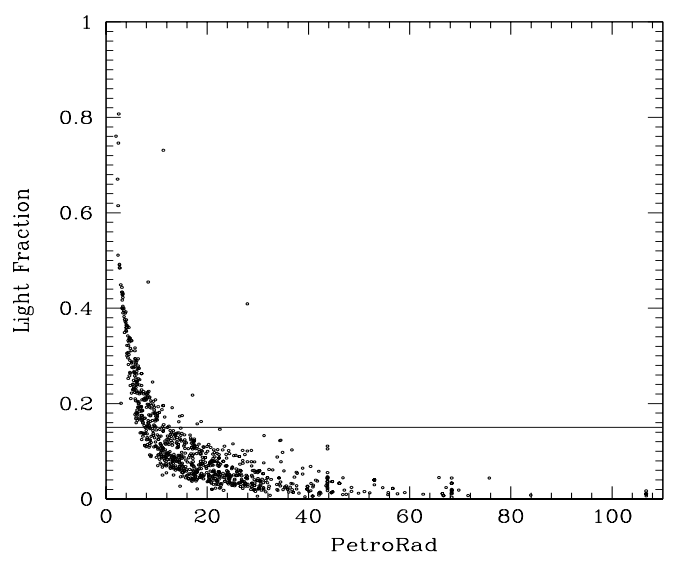

FIG. 2.- Relation between light fraction and Petrosian radius (in arcsec) in $\mathrm{r}$-band for the $980 \mathrm{SNe}$ host galaxies.

BPT diagram or not, as well as the total numbers of each type of SNe. We can see that among 169 SNe Ia hosts, 49 are SF galaxies, 49 are AGNs and 71 are Absorp galaxies.

\subsection{The comparative sample with low light fraction}

For those galaxies whose light fraction is lower than $15 \%$, we will take them as a comparative sample and perform a similar analysis. With the light fraction cut and further selecting criteria as described in Sect. 2.5, this comparative sample includes 689 galaxies. All the details about this part of galaxies will be given in Sect. [5.1. where we will show the difference between 213 samples and 689 samples.

\section{RELATIONS OF SOME PARAMETERS OF PROPERTIES} FOR THE 213 SUPERNOVA HOST GALAXIES

Some parameters associated with properties can indicate the evolutionary status and star-forming history of the galaxies. In this section, we plot some relations between properties of the 213 hosts for different types of $\mathrm{SNe}$ as shown in Fig. 5. The parameters describing sample galaxies are taken from the MPA/JHU database.

$\mathrm{D}_{n}(4000)$ : The break at $4000 \AA$ is a strong interruption in the optical spectrum. Two narrow continuum bands (3850-3950 and 4000-4100 A, narrower than the first definition by Bruzual A. (1983)), as introduced by 
TABLE 1

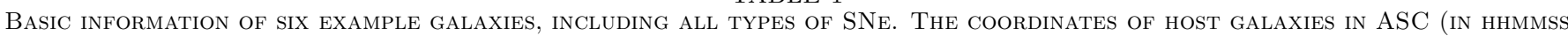

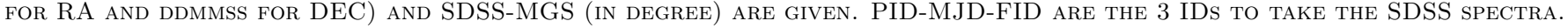

\begin{tabular}{|c|c|c|c|c|c|c|c|c|c|c|}
\hline No. & RA (ASC) & DEC (ASC) & RA (SDSS) & DEC (SDSS) & PID-MJD-FID & $\begin{array}{c}\begin{array}{c}\text { Petrion } \\
\text { radius }\end{array} \\
\end{array}$ & $\begin{array}{c}\text { light } \\
\text { fraction }\end{array}$ & redshift & Type & name of SN \\
\hline 111 & 105741 & 573648 & 164.42208862 & 57.61346436 & 0949-52427-109 & 6.375 & 0.193 & 0.080 & Ia & $2010 \mathrm{bg}$ \\
\hline 99 & 230548 & 141956 & 346.45358276 & 14.33142471 & $0742-52263-590$ & 7.429 & 0.209 & 0.108 & Ia & $2012 \mathrm{ff}$ \\
\hline 153 & 131523 & 462509 & 198.84954834 & 46.42040253 & $1461-53062-166$ & 8.732 & 0.162 & 0.056 & II & 2009 ct \\
\hline 130 & 163214 & 383920 & 248.05726624 & 38.65555954 & $1173-52790-537$ & 8.355 & 0.150 & 0.039 & II & 2012 ct \\
\hline 183 & 111229 & 312305 & 168.12567139 & 31.38496208 & $2092-53460-516$ & 6.309 & 0.176 & 0.027 & Ib & $2011 \mathrm{bp}$ \\
\hline 159 & 122450 & 082557 & 186.20838928 & 8.43370342 & $1626-53472-419$ & 7.143 & 0.174 & 0.090 & Ic & $2009 \mathrm{bh}$ \\
\hline
\end{tabular}

Note : Here we give only a few lines, with the meaning of all the columns. The full table will be available in the electronic version. The revised RA and DEC for some samples are given if we have them. The Nos. in first Column follow the full table. Here we present examples including all SNe Ia, II, Ib and Ic.

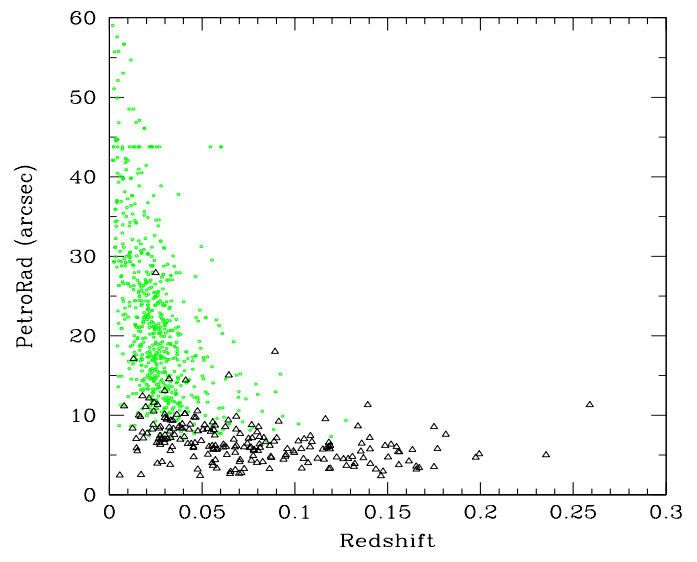

FIG. 3.- Relation between redshift and Petrosian radius (in arcsec) in r-band for the $980 \mathrm{SNe}$ host galaxies. The black triangles refer to the 213 galaxies having light fraction larger than $15 \%$, and the green circles refer to the 689 galaixes having lower light fraction.

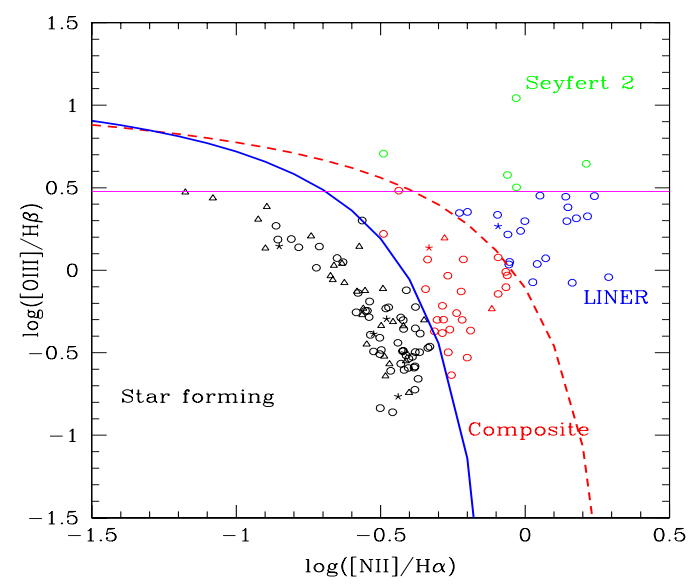

FIG. 4.- The BPT diagram for the 135 SNe hosts (among the 213 ) with good quality measurements of the four relevant emission lines. In the later sections, we will take SF as the starforming galaxies, and AGNs for the combined sample of Composites, Seyfert $2 \mathrm{~s}$ and LINERs. The three diagnostic lines are taken from Kauffmann et al. (2003a, the solid curve), Kewley et al, (2001, the dashed curve) and Shuder et al. (1981, the narrow solid horizonal line). Here the triangles refer to hosts of SNe II, the stars for hosts of SNe Ibc, and the circles refer to hosts of $\mathrm{SNe}$ Ia.

Balogh et al. (1999), were used to estimate this parameter. With increasing ages of the stellar populations of galaxies, the $\mathrm{D}_{n}(4000)$ values are increasing as well, indicating a larger fraction of older populations.

$\mathrm{H} \delta_{A}$ : A strong $\mathrm{H} \delta$ absorption line arises in galaxies
TABLE 2

THE NUMBers OF THE DIFFERENT TYPES OF SNE AMONG THE GALAXIES APPEARING OR NOT ON THE BPT DIAGRAM, AS WELL AS THE TOTAL NUMBERS.

\begin{tabular}{|c|c|c|c|c|c|}
\hline Samples & Galaxies & Total & $\mathrm{SN} \mathrm{I}$ & $\overline{\mathrm{SN} \mathrm{I}}$ & SN Ibc \\
\hline $\mathrm{SF}$ & Star-forming & 82 & 49 & 28 & 5 \\
\hline \multirow{3}{*}{ AGN } & Composite & 27 & 24 & 2 & 1 \\
\hline & LINER & 21 & 20 & 0 & 1 \\
\hline & Seyfert 2 & 5 & 5 & 0 & 0 \\
\hline Absorp & Absorp. \& WE & 78 & 71 & 4 & 3 \\
\hline Total & & 213 & 169 & 34 & 10 \\
\hline
\end{tabular}

Note : Here "Absorp." refers to absorption-line galaxies, and "WE" refers to weak emission-line galaxies that have not been detected all the four emission-lines in the BPT diagram.

that experienced a burst of star formation that ended about 0.1-1 Gyr ago. The peak of $\mathrm{H} \delta$ absorption occurs once hot $\mathrm{O}$ and $\mathrm{B}$ stars, which have weak intrinsic absorption, have terminated their evolution. The optical light from the galaxies is then dominated by late-B to early-F stars. Worthey \& Ottaviani (1997) defined an $\mathrm{H} \delta_{A}$ index, using a central bandpass (4083-4122A in the MPA/JHU database) bracketed by two pseudocontinuum band-passes.

The stellar masses of the SNe host galaxies are taken from Kauffmann et al. (2003b) and Gallazzi et al. (2005) The SFRs of them are taken from Brinchmann et al. (2004) (for the AGNs and absorption-line galaxies, they used the measured $\mathrm{D}_{n}(4000)$ value to estimate the SFRs and denote this as $\mathrm{SFR}_{d}$ ).

Figure 5 presents some relations between properties of the SNe hosts. The $\mathrm{D}_{n}(4000)$ vs. $\mathrm{H} \delta_{A}$, stellar mass vs. $\mathrm{D}_{n}(4000)$, stellar mass vs. SFRs and stellar mass vs. sSFRs are given from the first to fourth lines, respectively. On each line, the four panels show the relations when their hosts are SF, AGNs (composites, LINERs, Seyfert 2s), Absorp (absorption and weak emission line ones), and then all of 213 samples together with the SDSS MGS galaxies for background, respectively.

The interests of these results are as follows. First, since sample of our galaxies is large and they have good quality of spectral observations, the host galaxies of SNe of different types could be discussed following their BPT diagram, then the hosts as SFs, AGNs and Absorps are checked. Some of the previous studies often focus on one kinds of hosts, for example, Prieto et al. (2008) only study the SF ones which have metellicities $12+\log (\mathrm{O} / \mathrm{H})$ estimated from emission lines; Second, since we are considering hosts of SNe Ia, SNe II and SNe Ibc all together, we could compare the properties of hosts with different types of SNe, not only SNe Ia hosts or CC-SNe hosts. 
From both Table 2 and Figure 5, we notice that almost all the hosts of SNe II (the red triangles) are SF galaxies, and most of the SNe Ibc also occur in SF galaxies. A significant part of the SNe Ia (the black filled circles) occur in AGNs and the absorption (and weak emission) line galaxies. The rest part of SNe Ia occur in SF galaxies, which means their hosts have young stellar populations. These are consistent with the observation that $\mathrm{SNe}$ Ia can occur in all kinds of galaxies, from star-forming to passive cases.

Since our sample of host galaxies with different types of $\mathrm{SNe}$ can be classified as SF, AGN and Absorp galaxies, we could exhibit the distributions of some parameters of galaxies in these sub-groups. The top panels of Fig. 5 show that the star-forming (SF) host galaxies lie in the top-left region of the sample, showing they have experienced very recent star-forming activities and their young populations (with $\mathrm{D}_{n}(4000)<1.4$ ) are dominate. The AGNs (compsite, LINER and Seyfert 2) and absorptionline galaxies occupy the region with lower $\mathrm{H} \delta_{A}$ and higher $\mathrm{D}_{n}(4000)$, indicating they are dominated by old stellar populations. They are much more massive than the SF ones, $\log M / M_{\odot}>10$ generally. While many of the hosts as $\mathrm{SF}$ have both low (some down to $\log M / M_{\odot} \sim 8$ ) and high $\left(10<\log M / M_{\odot}<11\right)$ stellar masses.

For the all kinds of $\mathrm{SNe}(\mathrm{SNe} \mathrm{Ia}, \mathrm{SNe}$ II and $\mathrm{SNe} \mathrm{Ibc})$ hosts as SF, they all span a wide range of $\operatorname{mass}(8<$ $\left.\log M / M_{\odot}<11\right)$ and there is no obvious differences among them. The relations of stellar mass and SFRs show the increasing SFRs following increasing stellar masses in a wide range of mass of $\log M / M_{\odot} \sim 8-11$, for hosts of all types of SNe. In the bottom panels, the effect of mass was extracted from the SFRs, where we put the sample galaxies in the relations of sSFRs versus stellar mass. The discrepancy between the different $\mathrm{SNe}$ hosts is clear; most SNe II hosts are star-forming galaxies, very few are AGNs or weak emission line galaxies. The SNe Ia can explode in all kinds of galaxies. The hosts as AGNs and Absorption galaxies show an obvious discrepancy from the star-forming hosts. They are more massive and have low sSFRs.

It is interesting to compare the SNe host galaxies with the global SDSS MGS galaxies. The last column on each line in Fig. 5 show that the SNe host galaxies fall well within the regions of SDSS main galaxies. From this column, we see the discrepancy between the SNe II hosts and the SNe Ia hosts. The SNe Ia can explode from starforming to passive galaxies since their hosts fall within all the regions. Most of SNe II hosts locate in the regions of young spiral galaxies, suggesting they are dominated by young stellar populations. In a word, the host galaxies of SNe preferentially occupy some sub-regions of the diagrams depending on their host properties and $\mathrm{SNe}$ types.

Here we also notice that there are four SNe II hosts appearing on the figures of "Absorp" galaxies. We further check the images and spectra of these hosts. We find that three of them (ID: 1461-53062-166, 0377-52145$289,0387-51791-587)$ are the so-called weak emissionline galaxies. Their $\mathrm{H} \beta$ and/or [OIII] 5007 fluxes are under $3 \sigma$, while $\mathrm{H} \alpha$ and [NII]6583 can be measured from their spectra. By using the upper limits of the line fluxes, we roughly estimate their positions on the BPT diagram. The results show that they should belong to star-forming galaxies, but close to the lower end of [OIII $] 5007 / \mathrm{H} \beta$, suggesting they may be metal-rich galaxies. Their [OIII]5007 lines become too weak since there are many metal ions as coolant in metal-rich environment. There is one SNe II host (ID: 2586-54169-158) whose spectrum shows that it is a typical passive galaxy. But when we check its image, we find that there is a spiral galaxy which is very close to this SN II host. We think there may be some uncertainties when determining which galaxy is the host of this type II SNe, and this is beyond the research of this paper. There are still three SNe Ibc hosts appearing on "Absorp" galaxies and their situations are very similar with those four SNe II hosts. Two of them (ID: 0391-51782-442, 1626-53472-419) are weak emission-line galaxies. But for the other SN Ibc host (ID: 1337-52767-086), it should be an passive galaxies, from both image and spectrum. This is interesting and should be further studied in future.

\section{STELLAR POPULATIONS OF THE 213 HOST GALAXIES FROM SPECTRAL SYNTHESIS ANALYSIS}

To retrieve the stellar populations of SNe hosts, here we consider all the 213 sample galaxies as a representative sample to study and compare the properties of the hosts, i.e., the Type Ia, Type II and Type Ibc. Table 2 shows the corresponding numbers of host galaxies as 169, 34 and 10, respectively. Here we will obtain information about their detailed stellar population by fitting the full optical spectra using the spectral synthesis method, both on the continuum and absorption lines.

\subsection{Spectral synthesis method}

Spectral synthesis provides an efficient way to retrieve information on stellar populations of galaxies from observed spectra, which is a crucial step for a deeper understanding of galaxy formation and evolution. This is because galaxy spectra contain information on both the age and the metallicity distributions of their stars, which in turn reflects the star formation and chemical histories of the gallaxies.

We fit the spectral absorptions lines and continua of the sample galaxies to study their stellar populations by using the software Starlight ${ }^{8}$ (Cid Fernandes et al. 2005, 2007; Mateus et al. 2006; Asari et al. 2007; Chen et al. 2009). This software fits an observed spectrum $O_{\lambda}$ with a model $M_{\lambda}$ that adds up $N_{*}$ Simple Stellar Populations (SSPs) with different ages and metallicities from different stellar population synthesis models. A Gaussian distribution centered at velocity $v_{*}$ and broadened by $\sigma_{*} \bmod -$ els the line-of-sight stellar motions. The fit is carried out with the Metropolis scheme (Cid Fernandes et al. 2001), which searches for the minimum $\chi^{2}=\Sigma_{\lambda}\left[\left(O_{\lambda}-M_{\lambda}\right) \omega_{\lambda}\right]^{2}$, where $\omega_{\lambda}^{-1}$ is the error in $O_{\lambda}$ except for masked regions. Pixels that are more than $3 \sigma$ away from the rms $O_{\lambda}-M_{\lambda}$ are given zero weight by the parameter ' $c l i p^{\prime}$.

In the outputs of STARLIGHT, one of the most important parameters that traces the stellar population is the population vector $\vec{x}$. The component $x_{j}(j=$ $\left.1, \ldots, N_{*}\right)$ represents the fractional contribution of the SSP with age $t_{j}$ and metallicity $Z_{j}$ to the model flux at the normalization wavelength $\lambda_{0}=4020 \AA$. Another important parameter, the mass fraction $\mu_{j}$, has

\footnotetext{
8 http://www.starlight.ufsc.br
} 

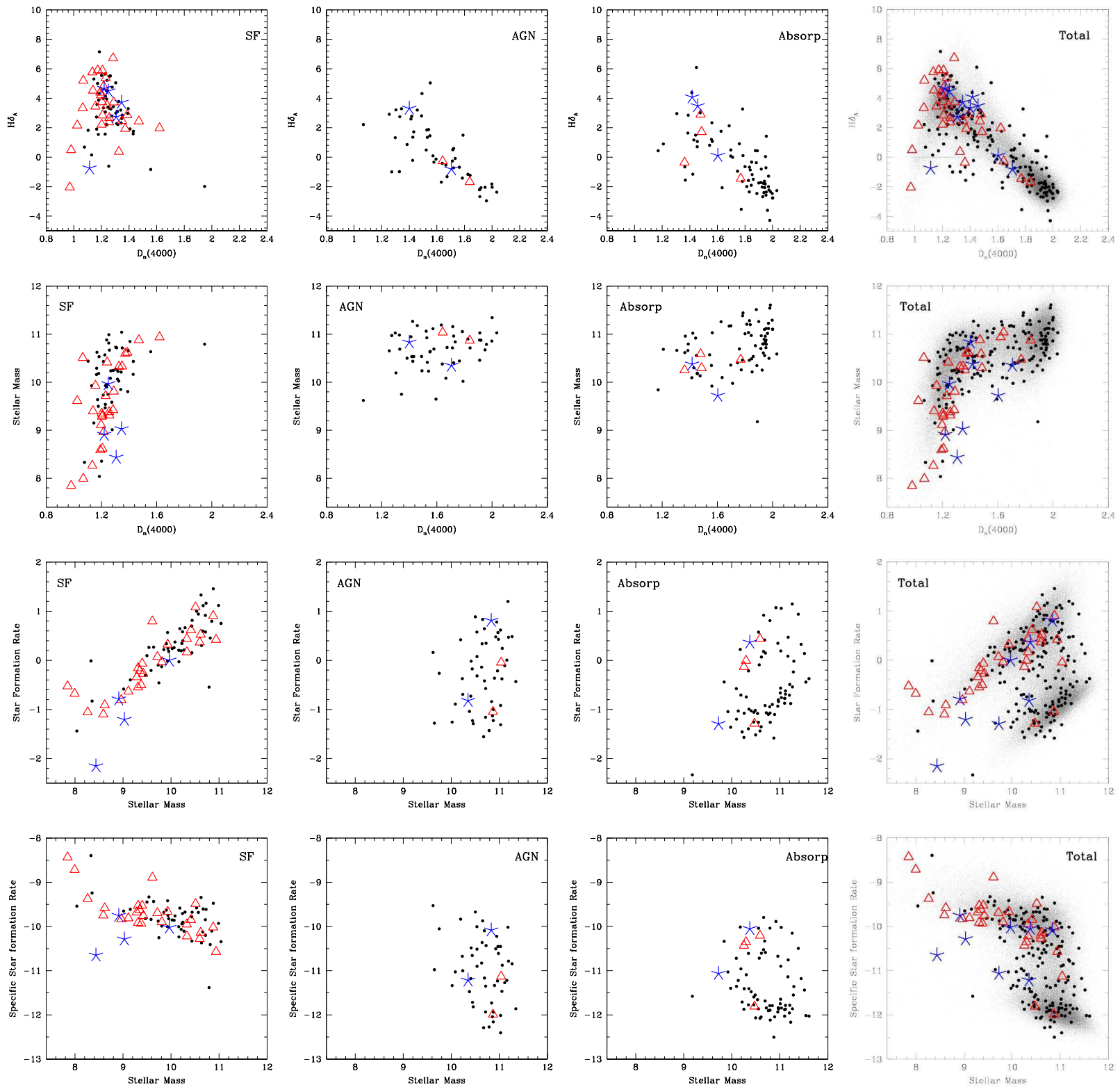

FIG. 5. - The relations among several property parameters for the $213 \mathrm{SNe}$ host galaxies: the $\mathrm{D}_{n}(4000)$ vs. $\mathrm{H} \delta_{A}$, and stellar mass vs. $\mathrm{D}_{n}(4000)$, stellar mass vs. SFR and sSFR on each line, respectively. The star-forming (SF) galaxies, AGNs (composite, LINER, Seyfert 2 ), absorption and weak emission line $(\operatorname{Absorp}(+\mathrm{WE}))$ galaxies and the complete sample of them (Total) are shown in panels from left to right. In all these panels, the red triangles refer to the hosts of SNe II, the blue stars for hosts of SNe Ibc, and the black filled circles for hosts of SNe Ia. These SNe host galaxies are compared with the SDSS main galaxy sample of galaxies (the dotted background) in the last panel of each line.

a similar meaning. For the uncertainties in the fitting results, Starlight group has carefully checked the reliability of this software by analyzing the "stellar populations" of fake galaxies made with known SSPs (see Fig.4 in Cid Fernandes et al. (2005), and Fig.1 in Cid Fernandes et al. (2004)). Cid Fernandes et al. (2005) presented their errorbars centered on the mean values obtained by fitting 20 realizations of each of 65 test galaxies. Their three condensed populations are recovered well by Starlight, with uncertainties smaller than 0.05 (young: $t<10^{8}$ ), 0.1 (intermediate: $10^{8}<t<10^{9}$ ) and 0.1 (old: $t>10^{9}$ ) for $\mathrm{S} / \mathrm{N}>10$.

In this work, the optical spectra of the SNe host galaxies were fit by using the Starlight code. We use 45 SSPs from Bruzual \& Charlot (2003) (BC03), including 15 dif- ferent ages from 1 Myr to 13 Gyr (i.e. 1, 3, 5, 10, 25, 40, 100, 280, 640, $900 \mathrm{Myr}$ and 1.4, 2.5, 5, 11, $13 \mathrm{Gyr}$ and 3 metallicities (i.e. $0.2,1$, and $2.5 Z_{\odot}$ ); the stellar evolutionary tracks of Padova 1994 (Alongi et al. 1993; Girardi et al. 1996); the Initial Mass Function (IMF) of Chabrier (2003); and the extinction law of Cardelli et al. (1989) with $R_{V}=3.1$. The Galactic extinctions are corrected with the reddening map of Schlegel et al. (1998), then shifted to the rest frame. The range of the spectra is from 3700 to $7800 \AA$ with a step of $1 \AA$ and normalized to the median flux in the 4010 to $4060 \AA$ region. During spectral synthesis fitting, we exclude the emission lines, the sky lines and four windows (5870$5905 \AA, 6845-6945 \AA, 7550-7725 \AA, 7165-7210 \AA$ ), as done in Chen et al. (2009, 2010). For LINERs and Seyfert 
$2 \mathrm{~s}$, a power law contribution has been added following Chen et al. (2010).

The Starlight code will result in a contribution to the percentage of each SSP at a given age and metallicity to the whole SED of the galaxy. To see the general trend, the SSPs are put in 3 bins: young populations with age $<0.2 \mathrm{Gyr}$, intermediate populations with age $0.2-2$ Gyr and old populations with age $>2$ Gyr (following Chen et al. (2010)). The stellar populations at three metallicities (i.e. $0.2,1$ and $2.5 Z_{\odot}$ ) are also obtained.

\subsection{The results from Starlight}

We have done the spectral synthesis analysis for all the 213 individual galaxies. In Fig. 6, we show an example of the fitting and results.

This figure consists of four parts of plots: the top left one displays the synthesis spectrum (red line), the observed spectrum (black line), and the error spectrum (blue line); the bottom left one shows the residual spectrum, where the green lines represent masked regions as given by the SDSS flag; the right panel shows the fractional contribution in light (top) and mass (bottom) from the 45 SSPs with different ages. We list the resulting six parameters in the top right corners, namely $\chi_{\lambda}{ }^{2}$, i.e. the reduced $\chi^{2}$; the mean relative difference between synthesis and observed spectra $\Delta_{\lambda}$; the $\mathrm{S} / \mathrm{N}$ in the region of 4730-4780 $\AA$; the $V$-band extinction; the velocity $v_{\star}$ and the velocity dispersion $\sigma_{\star}$.

In Fig. 7, we compare the stellar populations of hosts of different types of supernovae, where we separate the 213 sample galaxies into three groups: hosts of SNe Ia, SNe II and SNe Ibc, respectively. The top panel of Fig. 7 presents the KS test for the young populations $(<0.2$ Gyr) of the hosts of three types of supernovae. The middle panel of Fig. 7 presents the KS test for the metal-rich populations $\left(Z \sim 2.5 Z_{\odot}\right)$ of the hosts of three types of supernovae, and the bottom panel for the light-weighted mean ages $<\log _{*}>_{L}$ of the hosts of three types of supernovae.

The top panel of Fig. 7 shows that the hosts of SNe II have more young stellar populations than hosts of $\mathrm{SNe}$ Ia. It is difficult for us to distinguish the hosts of SNe Ibc from the hosts of both SNe II and SNe Ia, partly owing to the small number of $\mathrm{SNe}$ Ibc hosts. The possibilities of two hosts being drawn from the same distribution are $3.8 \times 10^{-5}$ (Ia-II), 0.17 (Ia-Ibc) and 0.42 (II-Ibc). We should also notice that there is still a fraction of SNe Ia hosts which have large young stellar populations, suggesting that SNe Ia can also explode in star forming galaxies. The middle panel shows that the hosts of SNe Ia have more metal-rich stellar population than hosts of SNe II. The differences between hosts of SNe Ibc and hosts of SNe Ia, as well as SNe II, are still not obvious. The possibilities of two hosts being drawn from the same distribution are $1.2 \times 10^{-4}$ (Ia-II), 0.56 (Ia-Ibc) and 0.15 (II-Ibc). The bottom panel shows that the hosts of SNe Ia have older ages than hosts of SNe II. Hosts of SNe Ibc are not distinguished from them obviously. The possibilities of two hosts being drawn from the same distribution are $3.8 \times 10^{-5}$ (Ia-II), 0.051 (Ia-Ibc) and 0.50 (II-Ibc).

From all 3 figures, we notice that the differences between SNe Ia hosts and SNe II hosts are significant, both for age and metallicities. The differences between the hosts of SNe Ibc and hosts of the other two types of SNe
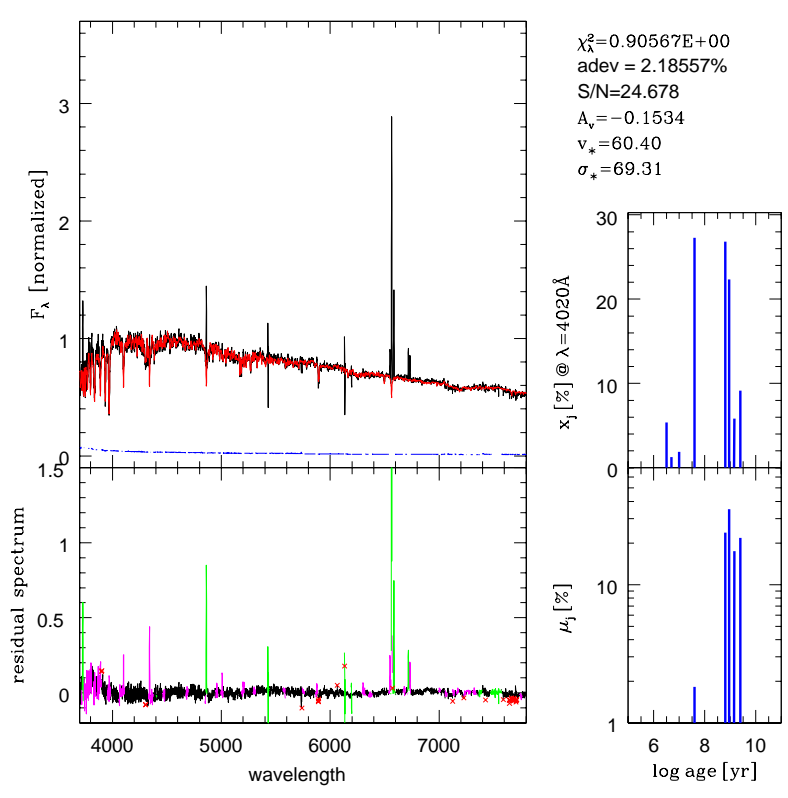

FIG. 6. - The spectral synthesis results for one example of SNe Ia host galaxies (PID, MJD, FID as 366, 52017, 485 respectively) by using Starlight with 45 SSPs from Bruzual \& Charlot (2003). We have at top left: the synthesis spectrum (red line), the observed spectrum (black line) and the error spectrum (blue line); at bottom left: the residual spectrum, the green lines representing masked regions as given by the SDSS flag; on the right: the contributed fractions of light (top) and mass (bottom) as a function of the 15 ages of SSPs. The resulting six parameters are listed in the top right corners. (Please see the online color version for more details.)

are not significant enough for us to get a strong conclusion.

\section{DISCUSSIONS}

\subsection{The comparative sample with low light fraction}

In the main part of this work, we present the properties of SNe host galaxies which have SDSS fiber observations covering at least $15 \%$ of the light from the galaxy as our main working sample. For the remaining part that has a lower light fraction, we take them as comparison sample as mentioned in Sect. 2.6. Their characteristic relations are given in Fig. 8 following Fig. 5, The sample galaxies are given in Table 3 following the same items in Table 2.

Table 3 shows that, in the 689 galaxies, the SFs are $50 \%, \mathrm{AGNs}$ are $41 \%$ and Absorption galaxies are $9 \%$. In the 213 galaxies shown in Table 2. the SFs are 38.5\%, AGNs are $24.9 \%$ and Absorption cases are $36.6 \%$. This means the comparative sample includes less Absorption cases as the hosts of SNe Ia. The increase of the AGN fraction can be understood since the nuclei region are covered in the fiber when targeting the large galaxies. This also shows the aperture effect of the fiber observations. Therefore, we believe the criterion of light fraction $>0.15$ is necessary to present the global properties of host galaxies of SNe.

For these 689 hosts from the comparative sample, we only show two sets of plots in Fig. 8, following Fig. 5 for the main working sample. As a result of the aperture effect, when compare Fig. 8 with Fig. 5, we can see some low SFRs case at a given stellar mass are added in the 689 galaxies, which makes the data more scattered. Owing to adding some large spiral galaxies, whose central regions 

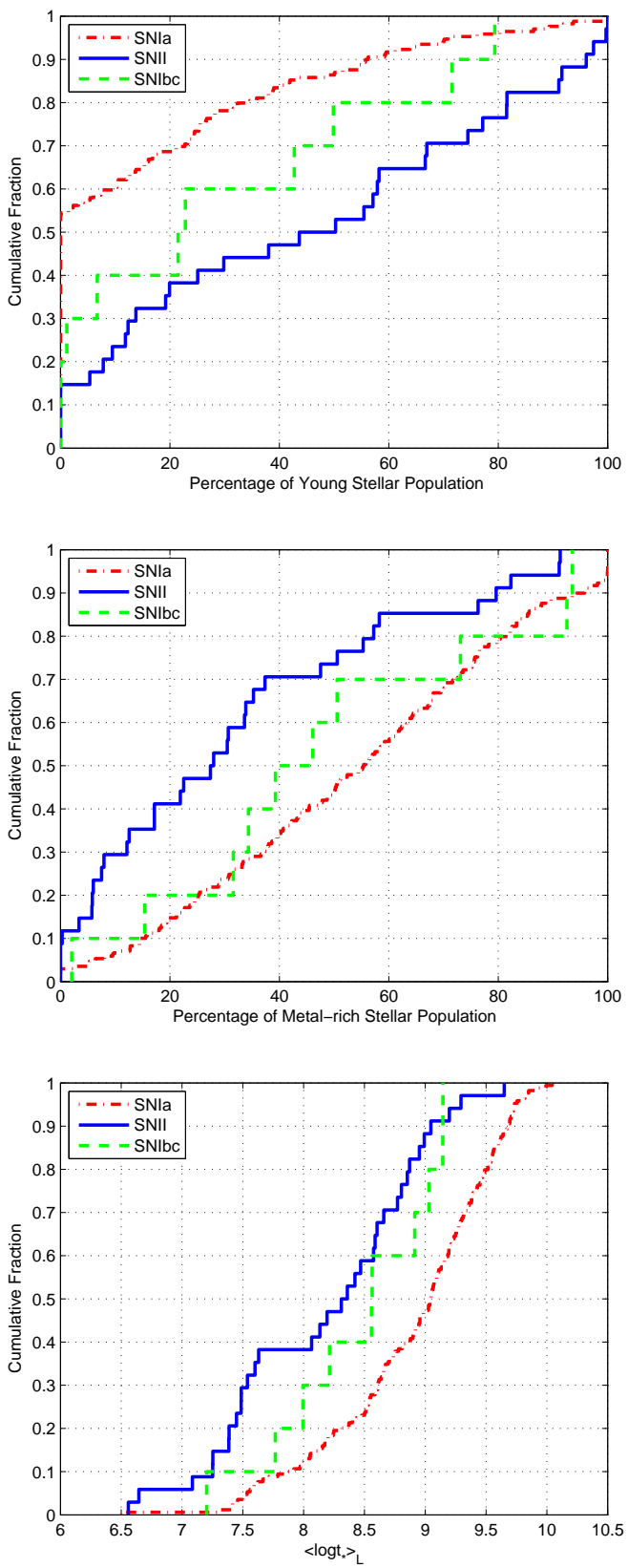

FIG. 7.- The cumulative fraction from the KS test for the stellar populations of the three types of supernovae in the 213 sample galaxies: (a) for the young population $(<0.2 \mathrm{Gyr})$, (b) for the metal rich population $\left(Z \sim 2.5 Z_{\odot}\right)$, (c) for the light-weighted mean ages $\left\langle\log _{*}\right\rangle_{L}$ of the three types of supernovae. The dot-dashed line is for hosts of SNe Ia, the solid line is for hosts of SNe II, and the dashed line is for hosts of $\mathrm{SNe} \mathrm{Ibc}$.

are just covered by SDSS, the SFRs of the 689 sample are lower than those of 213 samples. This phenomenon can be obviously seen when we compare the 689 sample with the SDSS main galaxies in the fourth column of Fig. 8, and compare with the 213 galaxies in Fig. 5 . The aperture effects are shown then.

Therefore, we believe the criterion of light fraction $>$ 0.15 is necessary to better present the global properties of host galaxies of SNe.

\subsection{The gas-phase metallicities and the aperture effect/bias}

TABLE 3

The Numbers of DIFFERENT TYPES OF SNE AMONG THE GALAXIES APPEARING OR NOT ON THE BPT DIAGRAM, AS WELL AS THE total numbers, FOR the 689 galaxies. The meanings are THE SAME AS IN FIG 5

\begin{tabular}{|c|c|c|ccc|}
\hline Samples & Galaxies & Total & SN Ia & SN II & SN Ibc \\
\hline SF & Star-forming & 345 & 114 & 177 & 54 \\
\hline \multirow{3}{*}{ AGN } & Composite & 135 & 66 & 54 & 15 \\
& LINER & 121 & 68 & 46 & 7 \\
& Seyfert 2 & 25 & 11 & 12 & 2 \\
\hline Absorp & Absorp. \& WE & 63 & 40 & 19 & 4 \\
\hline Total & & 689 & 299 & 308 & 82 \\
\hline
\end{tabular}

The metallicities and stellar masses are fundamental parameters to indicate the evolution status and history of galaxies. Therefore, it is worth obtaining the relation of stellar mass vs. metallicity (MZR) for the $\mathrm{SNe}$ host galaxies. This analysis is performed for both the 213 galaxies and the 689 comparison galaxies. Here, gasphase oxygen abundances are taken into account, thus only star forming galaxies are considered. There are 74 from the 213 and 314 from the 689 samples. Fig. 9 shows that the 74 galaxies locate closer to the MGS galaxies, but the 314 objects show about 0.1 dex higher $12+\log (\mathrm{O} / \mathrm{H})$ at a given stellar mass. The details are given as follows.

The top panels of Fig. 9] show the MZR of 74 SF galaxies (among 213 samples) and the KS test of the cumulative fraction of $12+\log (\mathrm{O} / \mathrm{H})$ for hosts of SNe Ia, II and Ibc. The hosts of SNe Ia are more metal-rich than the hosts of SNe II (Figs. 9a,b,c) generally. The lower panels of Fig. 9 are for the 314 SF galaxies (among 689 samples). Comparing the solid lines in Fig. 9r and Fig. 9F, it shows that these 314 galaxies are more metal-rich than the 74 galaxies in our main working sample, which shows the aperture effect clearly, namely, the fiber observations cover more in central parts for these 314 host galaxies, and thus they are more metal-rich than the 74 galaxies mentioned above at a given stellar mass.

When comparing Fig. 9a and Fig. 9d, we can see that the points and dashed line (the 2 order polynomial fit to the MZR for the 314 host galaxies) are generally 0.1 dex higher than the solid line (the 2 order polynomial fitting to the MZR of the 74 galaxies, which are shown in Fig. 9a), at a given stellar mass. The main galaxy sample from SDSS of Tremonti et al. (2004) are also added here as the dashed-line. We can see that the dashed-line is close to the solid line, which suggests the distribution of 74 galaxies is consistent with that of main galaxies. From Fig. 9b and Fig. 9p, for the comparison with the SDSS main galaxy sample as the background, it shows that the 74 galaxies are distributed nicely among the SDSS galaxies, but the 314 galaxies bias toward the more metal-rich region at a given stellar mass. These results are also the evidences that reducing the aperture bias is very necessary to present the global properties of SNe hosts.

In Fig. 9f, it is the KS test of the oxygen abundances of the 314 SNe host galaxies with a lower light fraction. The possibilities of SNe Ia hosts and SNe II hosts being drawn from the same distribution are 0.25 . This difference is less obvious than in Fig. 9k, in which the possibilities of SNe Ia hosts and SNe II hosts being drawn from the same distribution are 0.005 . We hold that the difference of significance is from the aperture bias, which we have 

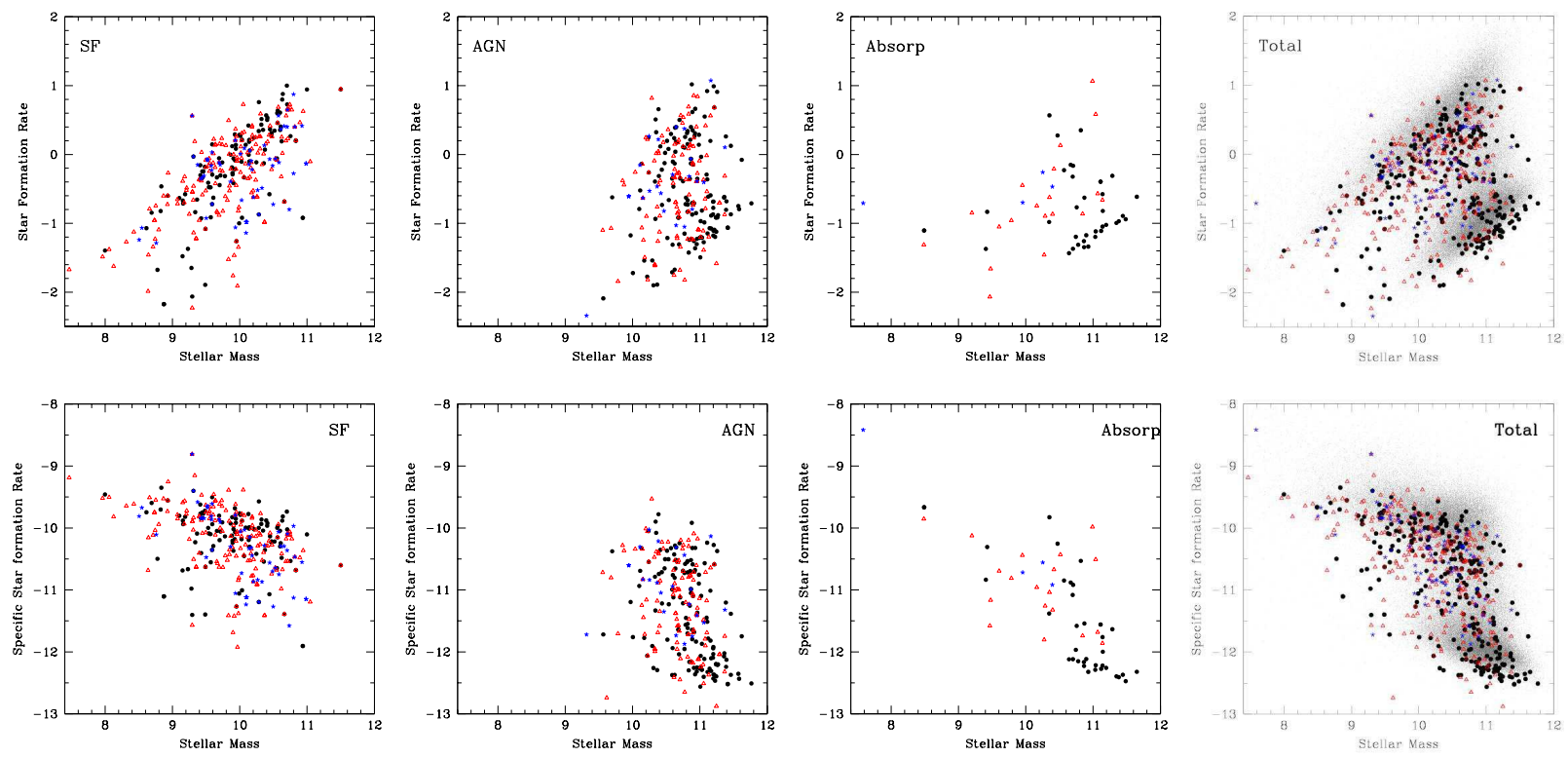

FIG. 8. - The relations of stellar masses vs. SFRs and sSFRs for the 689 comparison sample. The symbols are the same as in Fig. 5

discussed above.

The difference between SNe Ia hosts and SNe Ibc hosts are not obvious in Fig. 9c (significance as 0.9) perhaps part of the reason is the small number of the sample. In Fig. 9f, it shows that there are no significant differences between different SN types, and the KS possibilities of SNe Ibc hosts and SNe Ia hosts being drawn from the same distribution are 0.29 here. This result is similar to Fig.3 in Prieto et al. (2008), Prantzos \& Boissier (2003) and Boissier \& Prantzos (2009). This is understandable since both their samples and ours are focusing on star forming galaxies. This is a bit different from the results in our Sect.4.2, where not only star forming galaxies are considered, but also some AGNs and Absorption galaxies are included.

\section{CONCLUSIONS}

In this work, we selected $902(213+689)$ SNe of different types to study and compare the properties of their host galaxies. It is an improvement for such comparison studies since here both SNe Ia and CC-SNe (SNe II and $\mathrm{SNe} \mathrm{Ibc}$ ) are considered together, and we will consider the hosts as SFs, AGNs and Absorption galaxies rather than only star forming galaxies. The sample was obtained by cross-correlating the Asiago Supernova Catalog with the SDSS DR7 Main Galaxy Sample. In particular, we use a stricter criterion to select the sub-sample of 213 galaxies for detailed studies by requesting the 3 arcsec SDSS fiber observations covering at least $15 \%$ of the light of the whole galaxies, so that the spectra can represent the global properties of the whole galaxies. The remaining 689 galaxies with a lower light fraction of spectral observations are taken as a comparison sub-sample. Then the aperture effect/bias are shown clearly by comparing these two sub-samples. The sample includes Type Ia, Type II and Type Ibc SNe hosts, so we could compare the environments and properties of these different types of hosts at the same time.

Thanks to the SDSS for observing the high quality optical spectra and the MPA/JHU group for publishing the property parameters of the galaxies, we can then compare and study the sample galaxies in detailed stellar population analysis and in some interesting relations.

We summarize our results as follows.

1. We further classify the sample galaxies by their emission-line ratios on BPT diagram. Among the 213 sample galaxies, 135 of them can be plotted on the BPT diagram, including 82 star-forming (SF) galaxies and 53 AGNs (including composites, LINERs and Seyfert 2s). The other 78 cannot appear on BPT diagram as absorption (and weak emission) line galaxies (named as Absorp) (See Table2).

2. As shown in Fig. 5, almost all the type II SNe occur in star-forming (SF) galaxies, and only very few are in the AGNs and weak emission-line galaxies. Most of the $\mathrm{SNe} \mathrm{Ibc}$ are also in SF galaxies. The major part of the Type Ia SNe occur in AGNs and Absorp galaxies, and about one third in $\mathrm{SF}$ galaxies. The host galaxies as SF have a wide range of stellar mass, from $\log \left(\mathrm{M} / \mathrm{M}_{\odot}\right) \sim 8$ to 11 . However, the host galaxies as AGNs and Absorp galaxies are massive, mostly with $\log \left(\mathrm{M} / \mathrm{M}_{\odot}\right)>10$, and the Absorp host galaxies are even more massive, up to 11.6.

3 . When we put all these SNe host galaxies together in the relations of $\mathrm{D}_{n}(4000)$ vs. $\mathrm{H} \delta_{A}$, stellar mass vs. $\mathrm{D}_{n}(4000)$, stellar mass vs. SFRs and sSFRs, two groups for $\mathrm{SF}$ and a significant part of Absorp \& AGNs can been shown for the hosts, but the remaining part of the AGN \& Absorp is in the middle and even mixed with the corner of the SF galaxies. This is especially clear in the relation of stellar masses vs. SFRs and sSFRs as shown in Fig. 5. Thus, the SNe host galaxies fall well within the global SDSS sample, but preferentially occupy some sub-regions of the diagrams depending on the properties of their hosts.

4. The KS test of the cumulative fraction for stellar population analysis from spectral synthesis fitting 

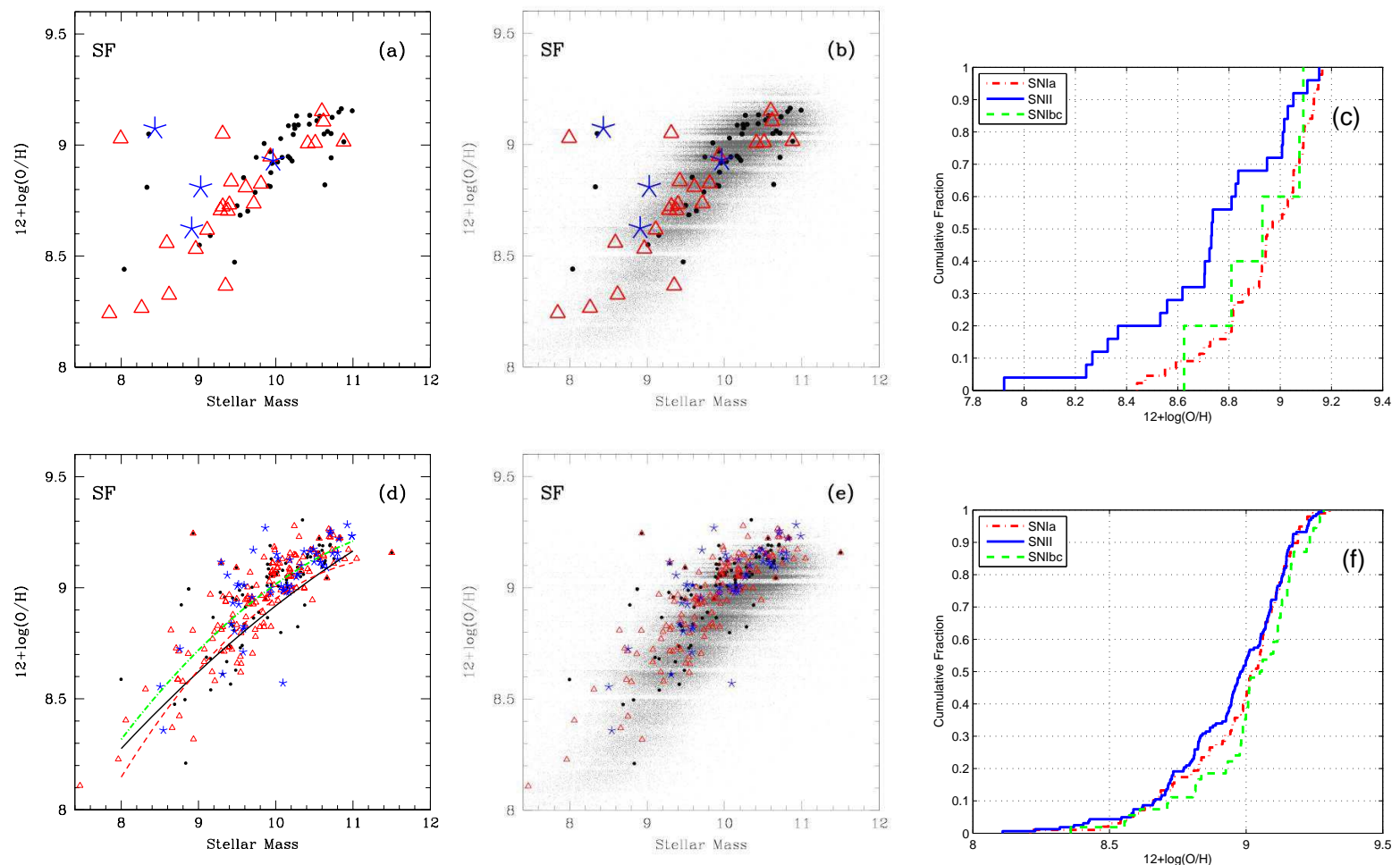

FIG. 9. - The mass-metallicity relations of the SNe host galaxies: the top panels for the 74 SF galaxies (among the 213 ones with higher light fraction than $15 \%$ in the SDSS fiber observations), and the bottom panels are for the 314 SF galaxies (among 689 ones that have a low light fraction). The triangles are hosts of SNe II, the stars for SNe Ibc and the filled circles for SNe Ia. In the KS test of the cumulative fraction, the solid line is for hosts of SNe II, the dashed line is for SNe Ibc and the dot-dashed line is for SNe Ia. In Fig.d, the thick dot-dashed line is for the polynomial fit for the 314 host galaxies, the solid line is for the 74 hosts, the objects in Fig.a, and the dashed line is for MGS of SDSS. The symbols are same as in Fig. 5 (Please see the online color version for details)

shows that the hosts of type II SNe have a younger stellar population and are younger than hosts of Type Ia SNe. The hosts of SNe Ia have more metalrich stellar pop- ulations and are more metal-rich than hosts of SNe II. The differences between the hosts of SNe Ibc and the hosts of other two types of $\mathrm{SNe}$ are not obvious.

5. The same relations of parameters describing properties have been made for the comparison sample with 689 galaxies. The AGN fraction of the sample is higher than that of the 213 objects since only nuclei region are covered in the fiber targeting the large galaxies.

6. The stellar mass-metallicity relations of the star forming galaxies in the two sub-samples are also presented. In the MZR, our main working sample of the galaxies with a higher light fraction of spectral observations are closer to the SDSS MGS galaxies, but the comparison sample is about 0.1 dex higher in $12+\log (\mathrm{O} / \mathrm{H})$ at a given stellar mass. This confirms that the aperture effect of spectral observations should be taken into account when we try to understand the properties of SN host galaxies. The KS test for the comparative sample
(Fig. 9f) shows that there are no significant differences between hosts of different SN types. We should keep in mind that only star forming galaxies are considered here.

In this work we have concentrated on the properties of the SNe hosts. However, we could not give more restrictions on the progenitors of supernovae, and the relations between the host properties and properties of the $\mathrm{SNe}$ themselves, such as the decline time, the stretch, the SNe peak luminosity or the SNe rates. These should be discussed in further studies.

We appreciate our referee who provided very admirable, constructive and helpful comments and suggestions, which helped to improve well our work. We also thank James Wicker for improving our English description in the text from the native language. We thank Zhanwen Han, Xiangdong Li, Tianmeng Zhang, Bo Wang, Xiaofeng Wang, Yan Gao for helpful discussions. This study is supported by the Natural Science Foundation of China under grants Nos. 10933001, 11273026, 11273011, 11178013 and the National Basic Research Program of China (973 program) No. 2007CB815104/06.

\section{REFERENCES}

Abazajian, K., Adelman-McCarthy, J. K., Agüeros, M. A., et al. 2003, AJ, 126, 2081

Abazajian, K., Adelman-McCarthy, J. K., Agüeros, M. A., et al. 2004, AJ, 128, 502

Alongi, M., Bertelli, G., Bressan, A., et al. 1993, A\&AS, 97, 851
Anderson, J. P., \& James, P. A. 2009, MNRAS, 399, 559

Asari, N. V., Cid Fernandes, R., Stasińska, G., et al. 2007, MNRAS, 381,263

Baldwin, J. A., Phillips, M. M., \& Terlevich, R. 1981, PASP, 93, 5 
Balogh, M. L., Morris, S. L., Yee, H. K. C., Carlberg, R. G., \& Ellingson, E. 1999, ApJ, 527, 54

Barbon, R., Buondí, V., Cappellaro, E., \& Turatto, M. 1999, A\&AS, 139, 531

Barbon, R., Cappellaro, E., Ciatti, F., Turatto, M., \& Kowal, C. T. 1984, A\&AS, 58, 735

Barbon, R., Cappellaro, E., \& Turatto, M. 1989, A\&AS, 81, 421

Boissier, S., \& Prantzos, N. 2009, A\&A, 503, 137

Brinchmann, J., Charlot, S., White, S. D. M., et al. 2004, MNRAS, 351,1151

Bruzual A., G. 1983, ApJ, 273, 105

Bruzual, G., \& Charlot, S. 2003, MNRAS, 344, 1000

Cappellaro, E., Evans, R., \& Turatto, M. 1999, A\&A, 351, 459

Cardelli, J. A., Clayton, G. C., \& Mathis, J. S. 1989, ApJ, 345, 245

Chabrier, G. 2003, PASP, 115, 763

Chandrasekhar, S. 1931, ApJ, 74, 81

Chen, X. Y., Liang, Y. C., Hammer, F., Zhao, Y. H., \& Zhong, G. H. 2009, A\&A, 495, 457

Chen, X. Y., Liang, Y. C., Hammer, F., et al. 2010, A\&A, 515, A101

Cid Fernandes, R., Sodré, L., Schmitt, H. R., \& Leão, J. R. S. 2001, MNRAS, 325, 60

Cid Fernandes, R., González Delgado, R. M., Schmitt, H., et al. 2004, ApJ, 605, 105

Cid Fernandes, R., González Delgado, R. M., Storchi-Bergmann, T., Martins, L. P., \& Schmitt, H. 2005, MNRAS, 356, 270

Cid Fernandes, R., Asari, N. V., Sodré, L., et al. 2007, MNRAS, 375, L16

Cooper, M. C., Newman, J. A., \& Yan, R. 2009, ApJ, 704, 687

D'Andrea, C. B., Sako, M., Dilday, B., et al. 2010, ApJ, 708, 661

della Valle, M., \& Livio, M. 1994, ApJ, 423, L31

Filippenko, A. V. 1997, ARA\&A, 35, 309

Frieman, J. A., Bassett, B., Becker, A., et al. 2008, AJ, 135, 338

Gallagher, J. S., Garnavich, P. M., Berlind, P., et al. 2005, ApJ, 634,210

Gallagher, J. S., Garnavich, P. M., Caldwell, N., et al. 2008, ApJ, 685,752

Gallazzi, A., Charlot, S., Brinchmann, J., White, S. D. M., \& Tremonti, C. A. 2005, MNRAS, 362, 41

Girardi, L., Bressan, A., Chiosi, C., Bertelli, G., \& Nasi, E. 1996, A\&AS, 117, 113

Habergham, S. M., Anderson, J. P., \& James, P. A. 2010, ApJ, 717,342

Hamuy, M., Phillips, M. M., Maza, J., et al. 1995, AJ, 109, 1

Hamuy, M., Phillips, M. M., Suntzeff, N. B., et al. 1996, AJ, 112, 2398
Hamuy, M., Trager, S. C., Pinto, P. A., et al. 2000, AJ, 120, 1479

Hamuy, M., Maza, J., Pinto, P. A., et al. 2002, AJ, 124, 417

Hakobyan, A. A., Adibekyan, V. Z., Aramyan, L. S., et al. 2012, A\&A, 544, A81

Han, D.-H., Park, C., Choi, Y.-Y., \& Park, M.-G. 2010, ApJ, 724, 502

Iben, I., Jr., \& Tutukov, A. V. 1984, ApJS, 54, 335

Kauffmann, G., Heckman, T. M., Tremonti, C., et al. 2003, MNRAS, 346, 1055

Kauffmann, G., Heckman, T. M., White, S. D. M., et al. 2003, MNRAS, 341, 33

Kelly, P. L., \& Kirshner, R. P. 2012, ApJ, 759, 107

Kewley, L. J., Dopita, M. A., Sutherland, R. S., Heisler, C. A., \& Trevena, J. 2001, ApJ, 556, 121

Lampeitl, H., Nichol, R. C., Seo, H.-J., et al. 2010, MNRAS, 401, 2331

Liang, Y. C., Zhong, G. H., Hammer, F., et al. 2010, MNRAS, 409, 213

Mateus, A., Sodré, L., Cid Fernandes, R., et al. 2006, MNRAS, 370,721

Neill, J. D., Sullivan, M., Howell, D. A., et al. 2009, ApJ, 707, 1449 Nomoto, K. 1982, ApJ, 253, 798

Oemler, A., Jr., \& Tinsley, B. M. 1979, AJ, 84, 985

Prantzos, N., \& Boissier, S. 2003, A\&A, 406, 259

Prieto, J. L., Stanek, K. Z., \& Beacom, J. F. 2008, ApJ, 673, 999

Schlegel, D. J., Finkbeiner, D. P., \& Davis, M. 1998, ApJ, 500, 525

Shuder, J. M., \& Osterbrock, D. E. 1981, ApJ, 250, 55

Smartt, S. J. 2009, ARA\&A, 47, 63

Stoughton, C., Lupton, R. H., Bernardi, M., et al. 2002, AJ, 123, 485

Strauss, M. A., Weinberg, D. H., Lupton, R. H., et al. 2002, AJ, 124,1810

Tremonti, C. A., Heckman, T. M., Kauffmann, G., et al. 2004, ApJ, 613,898

Turatto, M. 2003, in Supernovae and GRBs, v. 598 of Lecture Notes in Physics, ed. K. Weiler (Springer-Verlag), 21

van den Bergh, S. 1990, PASP, 102, 1318

Webbink, R. F. 1984, ApJ, 277, 355

Whelan, J., \& Iben, I., Jr. 1973, ApJ, 186, 1007

Williams, K. A., Bolte, M., \& Koester, D. 2009, ApJ, 693, 355

Worthey, G., \& Ottaviani, D. L. 1997, ApJS, 111, 377

York, D. G., Adelman, J., Anderson, J. E., Jr., et al. 2000, AJ, 120, 1579

Zheng, C., Romani, R. W., Sako, M., et al. 2008, AJ, 135, 1766 
TABLE 4

ON-Line DATA: BASIC INFORMATION OF THE SAMPLE GALAXiES.

\begin{tabular}{|c|c|c|c|c|c|c|c|c|c|c|}
\hline No. & RA (ASC) & DEC (ASC) & RA (SDSS) & DEC (SDSS) & PID-MJD-FID & $\begin{array}{c}\text { Petrion } \\
\text { radius }\end{array}$ & $\begin{array}{c}\text { light } \\
\text { fraction }\end{array}$ & redshift & Type & name of SN \\
\hline 1 & 101800 & -000158 & 154.50199890 & -0.03280395 & $0271-51883-171$ & 2.658 & 0.490 & 0.065 & Ia & $2000 \mathrm{fx}$ \\
\hline 2 & 111610 & -001139 & 169.04255676 & -0.19220079 & $0279-51984-180$ & 3.778 & 0.389 & 0.156 & Ia & $1996 \mathrm{R}$ \\
\hline 3 & 112225 & 011121 & 170.60607910 & 1.18944538 & $0280-51612-409$ & 6.564 & 0.182 & 0.029 & Ia & $1997 \mathrm{bz}$ \\
\hline 4 & 124733 & 000557 & 191.88919067 & 0.09919899 & 0291-51928-076 & 3.277 & 0.428 & 0.086 & Ia & $2001 \mathrm{kk}$ \\
\hline 5 & 133955 & 005215 & 204.98309326 & 0.87136489 & $0299-51671-380$ & 2.672 & 0.491 & 0.069 & Ia & 2005 ac \\
\hline 6 & 160713 & -000449 & 241.80650330 & -0.07878780 & 0344-51693-039 & 9.616 & 0.179 & 0.031 & II & 2001 ax \\
\hline 7 & 173228 & 560425 & 263.11889648 & 56.07372665 & $0358-51818-181$ & 4.720 & 0.290 & 0.122 & Ia & $2000 \mathrm{gb}$ \\
\hline 8 & 172822.703 & 573239.00 & 262.09924316 & 57.54539871 & $0358-51818-364$ & 7.019 & 0.184 & 0.028 & II & $2004 \mathrm{eb}$ \\
\hline 9 & 172611 & 591831 & 261.54681396 & 59.30865479 & 0366-52017-485 & 7.421 & 0.225 & 0.027 & Ia & 2009 ia \\
\hline 10 & 223507 & -010637 & 338.78216553 & -1.10929883 & $0377-52145-289$ & 5.047 & 0.222 & 0.095 & II & $2007 \mathrm{qv}$ \\
\hline 11 & 224142 & -000812 & 340.42520142 & -0.13693197 & $0377-52145-594$ & 3.322 & 0.430 & 0.057 & Ia & 2006 py \\
\hline 12 & 224459 & -010023 & 341.24508667 & -1.00638306 & 0378-52146-135 & 4.531 & 0.302 & 0.128 & Ia & $2006 \mathrm{nd}$ \\
\hline 13 & 225942 & -000049 & 344.92785645 & -0.01355672 & $0380-51792-192$ & 5.915 & 0.233 & 0.045 & Ia & $2005 \mathrm{ku}$ \\
\hline 14 & 231154 & -003441 & 347.97653198 & -0.57908654 & 0381-51811-106 & 9.227 & 0.245 & 0.091 & Ia & $2007 \mathrm{pd}$ \\
\hline 15 & 231729 & 002545 & 349.37374878 & 0.42967373 & $0382-51816-486$ & 3.337 & 0.403 & 0.119 & Ia & $2005 \mathrm{fh}$ \\
\hline 16 & 232640 & -005024 & 351.66729736 & -0.84061658 & 0383-51818-057 & 4.148 & 0.300 & 0.082 & Ia & 2006 fy \\
\hline 17 & 232439 & -004306 & 351.16223145 & -0.71791536 & 0383-51818-134 & 6.191 & 0.246 & 0.148 & Ia & 2006 ju \\
\hline 18 & 232447 & 005640 & 351.19976807 & 0.94429779 & $0383-51818-445$ & 6.072 & 0.289 & 0.118 & Ia & $2007 \mathrm{lv}$ \\
\hline 19 & 232749 & 002726 & 351.95654297 & 0.45790046 & $0383-51818-581$ & 5.753 & 0.177 & 0.118 & II & $2007 \mathrm{pg}$ \\
\hline 20 & 232807 & 005122 & 352.03250122 & 0.85817730 & 0383-51818-618 & 6.188 & 0.272 & 0.119 & Ia & 2006 ol \\
\hline 21 & 233947 & 001218 & 354.94631958 & 0.20583645 & $0385-51877-427$ & 6.176 & 0.186 & 0.068 & Ia & $2006 \mathrm{hq}$ \\
\hline 22 & 235420 & -005503 & 358.58627319 & -0.91723460 & 0386-51788-002 & 7.028 & 0.222 & 0.105 & Ia & $2007 \mathrm{om}$ \\
\hline 23 & 000348 & 002131 & 0.95135558 & 0.35961452 & $0387-51791-587$ & 5.300 & 0.229 & 0.099 & II & $2007 \mathrm{nx}$ \\
\hline 24 & 001124 & 004209 & 2.85239959 & 0.70202297 & 0388-51793-584 & 5.126 & 0.276 & 0.199 & Ia & $2006 \mathrm{jz}$ \\
\hline 25 & 001641 & -002528 & 4.17437315 & -0.42516112 & $0389-51795-151$ & 4.547 & 0.335 & 0.104 & Ia & $2006 \mathrm{fz}$ \\
\hline 26 & 002635 & -001807 & 6.64762497 & -0.30328667 & 0390-51900-033 & 5.012 & 0.310 & 0.235 & Ia & $2006 \mathrm{ff}$ \\
\hline 27 & 002137 & -010035 & 5.40788269 & -1.01061416 & 0390-51900-133 & 6.640 & 0.250 & 0.084 & Ia & 2006 er \\
\hline 28 & 002000 & -003729 & 5.00321960 & -0.62535346 & 0390-51900-237 & 5.332 & 0.240 & 0.067 & II & $2006 \mathrm{fq}$ \\
\hline 29 & 002244 & -002844 & 5.68314219 & -0.47940356 & 0391-51782-316 & 5.992 & 0.180 & 0.108 & Ia & $2007 \mathrm{px}$ \\
\hline 30 & 002741 & 011359 & 6.92455101 & 1.23239827 & $0391-51782-442$ & 8.554 & 0.153 & 0.080 & Ib & $2007 \mathrm{qx}$ \\
\hline 31 & 004900 & -001926 & 12.25123310 & -0.32327026 & 0393-51794-034 & 5.384 & 0.229 & 0.156 & Ia & $2007 \mathrm{lr}$ \\
\hline 32 & 004624 & 000012 & 11.60083675 & 0.00237388 & 0393-51794-106 & 9.534 & 0.186 & 0.116 & Ia & $2006 \mathrm{gf}$ \\
\hline 33 & 005252 & -000431 & 13.21838474 & -0.07436865 & 0394-51913-177 & 4.455 & 0.307 & 0.115 & II & $1996 \mathrm{bg}$ \\
\hline 34 & 004909 & 003549 & 12.28801918 & 0.59661305 & 0394-51913-389 & 5.722 & 0.263 & 0.115 & Ia & 2007 lc \\
\hline 35 & 005924 & 000009 & 14.85048771 & 0.00266656 & $0395-51783-511$ & 6.059 & 0.226 & 0.062 & Ia & 2005 ho \\
\hline 36 & 011026 & -010404 & 17.60987854 & -1.06876671 & 0397-51794-206 & 6.037 & 0.244 & 0.154 & II & $2006 \mathrm{gd}$ \\
\hline 37 & 011058 & 001634 & 17.74193001 & 0.27615604 & 0397-51794-477 & 2.925 & 0.449 & 0.065 & Ia & $2005 \mathrm{kt}$ \\
\hline 38 & 011357 & 002218 & 18.48825455 & 0.37141645 & $0397-51794-550$ & 6.034 & 0.224 & 0.045 & Ia & $2006 \mathrm{hx}$ \\
\hline 39 & 011337 & 002525 & 18.40657425 & 0.42374301 & 0397-51794-551 & 9.715 & 0.163 & 0.046 & Ia & 2006 ne \\
\hline 40 & 011612 & 004731 & 19.05294991 & 0.79055309 & 0398-51789-362 & 6.201 & 0.204 & 0.086 & Ia & $2005 \mathrm{gb}$ \\
\hline 41 & 011643 & 004740 & 19.18280983 & 0.79359138 & 0398-51789-378 & 5.974 & 0.159 & 0.076 & Ia & 2005 ir \\
\hline 42 & 011502 & 001542 & 18.75957489 & 0.26294789 & $0398-51789-440$ & 8.026 & 0.192 & 0.047 & Ia & $2004 \mathrm{hu}$ \\
\hline 43 & 012314 & -001946 & 20.81234741 & -0.33023268 & 0399-51817-191 & 8.780 & 0.174 & 0.076 & $\mathrm{Ib}$ & 2006 jo \\
\hline 44 & 012137 & 002452 & 20.40770721 & 0.41437322 & 0399-51817-349 & 3.564 & 0.394 & 0.131 & Ia & $2006 \mathrm{fv}$ \\
\hline 45 & 013723 & -001843 & 24.34909821 & -0.31172231 & $0400-51820-023$ & 5.707 & 0.310 & 0.056 & Ia & $2007 \mathrm{ol}$ \\
\hline 46 & 013441 & -003619 & 23.67431831 & -0.60421115 & $0400-51820-164$ & 4.035 & 0.363 & 0.079 & Ia & $2005 \mathrm{js}$ \\
\hline 47 & 013936 & -004531 & 24.90002060 & -0.75794107 & 0401-51788-284 & 4.233 & 0.363 & 0.161 & Ia & $2005 \mathrm{fa}$ \\
\hline 48 & 015236 & -001331 & 28.15243721 & -0.22807825 & 0402-51793-071 & 5.786 & 0.289 & 0.176 & Ia & $2002 \mathrm{gp}$ \\
\hline 49 & 015020 & -002413 & 27.58425140 & -0.40386590 & 0402-51793-179 & 3.675 & 0.348 & 0.127 & Ia & $2007 \mathrm{rc}$ \\
\hline 50 & 015840 & -001456 & 29.66862106 & -0.24855912 & 0403-51871-110 & 7.308 & 0.160 & 0.080 & II & 200711 \\
\hline 51 & 015654 & -010649 & 29.22780800 & -1.11471581 & 0403-51871-256 & 8.867 & 0.205 & 0.043 & Ia & $2002 \mathrm{gn}$ \\
\hline 52 & 015358 & -000533 & 28.49486351 & -0.09352394 & 0403-51871-307 & 6.715 & 0.170 & 0.088 & Ia & $2007 \mathrm{rj}$ \\
\hline 53 & 020232 & -010521 & 30.63646698 & -1.08993196 & 0404-51812-285 & 4.636 & 0.258 & 0.136 & Ia & $2007 \mathrm{jw}$ \\
\hline 54 & 020416 & 003911 & 31.07067490 & 0.65270579 & 0404-51812-372 & 7.091 & 0.170 & 0.075 & II & $2001 \mathrm{im}$ \\
\hline 55 & 020335 & 004710 & 30.89647484 & 0.78468317 & 0404-51812-373 & 6.329 & 0.191 & 0.061 & II & $2007 \mathrm{hw}$ \\
\hline 56 & 020503 & 001028 & 31.26476669 & 0.17514242 & 0404-51812-493 & 7.137 & 0.186 & 0.077 & Ia & $2007 \mathrm{mn}$ \\
\hline 57 & 020719 & 011507 & 31.82991409 & 1.25202501 & 0404-51812-565 & 8.535 & 0.197 & 0.174 & Ia & 2006 ia \\
\hline 58 & 021802 & -003332 & 34.50863647 & -0.55963063 & 0405-51816-066 & 3.197 & 0.400 & 0.143 & Ia & 2004 ia \\
\hline 59 & 022824 & 001104 & 37.09963226 & 0.18599379 & 0406-51869-633 & 3.573 & 0.382 & 0.165 & Ia & $2007 \mathrm{ok}$ \\
\hline 60 & 023759 & -010139 & 39.49538803 & -1.02751327 & 0408-51821-201 & 5.465 & 0.248 & 0.135 & Ia & $2001 \mathrm{eu}$ \\
\hline 61 & 023526 & 010429 & 38.86076736 & 1.07453907 & 0408-51821-337 & 4.446 & 0.321 & 0.093 & Ia & 2005 je \\
\hline 62 & 025229 & -010822 & 43.12130737 & -1.13941860 & $0410-51816-247$ & 6.460 & 0.203 & 0.136 & Ia & $2007 \mathrm{qr}$ \\
\hline 63 & 030702 & -000040 & 46.76201248 & -0.01128918 & 0411-51817-072 & 4.023 & 0.358 & 0.107 & Ia & 2004 il \\
\hline 64 & 025953 & 010938 & 44.97357178 & 1.16005981 & 0411-51817-322 & 6.006 & 0.178 & 0.072 & Ia & $2007 \mathrm{jd}$ \\
\hline 65 & 030522 & 005130 & 46.34433365 & 0.85972154 & 0411-51817-571 & 6.605 & 0.220 & 0.118 & Ia & $2005 \mathrm{fv}$ \\
\hline 66 & 030851 & -011024 & 47.21431732 & -1.17334652 & $0412-52258-210$ & 4.517 & 0.359 & 0.126 & Ia & $2001 \mathrm{kl}$ \\
\hline 67 & 032331 & 003960 & 50.88061905 & 0.66727877 & 0413-51929-621 & 3.924 & 0.392 & 0.132 & Ia & $2007 \mathrm{mi}$ \\
\hline 68 & 033012 & -005828 & 52.55371475 & -0.97447813 & $0415-51810-281$ & 6.976 & 0.164 & 0.067 & Ia & 2005 if \\
\hline 69 & 033602 & 010617 & 54.00661087 & 1.10475719 & $0415-51810-571$ & 10.190 & 0.178 & 0.040 & Ia & $2007 \mathrm{jh}$ \\
\hline 70 & 033602 & 010617 & 54.00661087 & 1.10475719 & $0415-51810-571$ & 10.190 & 0.178 & 0.040 & Ia & $2010 \mathrm{kf}$ \\
\hline 71 & 033942 & 010532 & 54.92742920 & 1.09268486 & 0416-51811-411 & 7.585 & 0.206 & 0.181 & Ia & 2007 hy \\
\hline 72 & 034044.4 & 010323.84 & 55.18504715 & 1.05656338 & 0416-51811-416 & 10.503 & 0.181 & 0.023 & Ia & $2010 \mathrm{jf}$ \\
\hline 73 & 034310 & 000608 & 55.79183960 & 0.10396237 & 0416-51811-514 & 4.798 & 0.263 & 0.130 & Ia & 2007 ia \\
\hline
\end{tabular}


TABLE 4

-CONTINUED

\begin{tabular}{|c|c|c|c|c|c|c|c|c|c|c|}
\hline No. & RA (ASC) & DEC (ASC) & RA (SDSS) & DEC (SDSS) & PID-MJD-FID & $\begin{array}{l}\text { Petrion } \\
\text { radius }\end{array}$ & $\begin{array}{c}\text { light } \\
\text { fraction }\end{array}$ & redshift & Type & name of SN \\
\hline 74 & 010905 & 144516 & 17.27483749 & 14.75579071 & $0422-51811-499$ & 8.377 & 0.197 & 0.038 & $\mathrm{Ib}$ & $2008 \mathrm{fs}$ \\
\hline 75 & 080313 & 473650 & 120.80256653 & 47.61380005 & $0438-51884-462$ & 5.947 & 0.284 & 0.117 & Ia & $2000 \mathrm{fy}$ \\
\hline 76 & 092229 & 575429 & 140.62144470 & 57.90814209 & $0452-51911-319$ & 8.549 & 0.153 & 0.062 & Ia & $2001 \mathrm{kj}$ \\
\hline 77 & 022814 & -083632 & 37.06108475 & -8.60764790 & $0454-51908-520$ & 7.191 & 0.170 & 0.140 & II & $2007 \mathrm{tn}$ \\
\hline 78 & 022904 & -082414 & 37.26552200 & -8.40379238 & 0454-51908-559 & 3.933 & 0.369 & 0.140 & Ia & $2000 \mathrm{ga}$ \\
\hline 79 & 031304.193 & -082354.24 & 48.26750946 & -8.39855385 & $0459-51924-130$ & 10.139 & 0.173 & 0.029 & Ia & $2012 \mathrm{eu}$ \\
\hline 80 & 091138 & -004254 & 137.90991211 & -0.71499681 & $0472-51955-247$ & 4.138 & 0.305 & 0.070 & Ia & $2001 \mathrm{~km}$ \\
\hline 81 & 095153 & 010606 & 147.97111511 & 1.10159636 & $0480-51989-024$ & 5.044 & 0.284 & 0.063 & Ia & $2001 \mathrm{kp}$ \\
\hline 82 & 141058 & 645051 & 212.74302673 & 64.84748840 & 0498-51984-102 & 5.747 & 0.294 & 0.140 & Ia & 2001 ko \\
\hline 83 & 132059 & 033556 & 200.24960327 & 3.59741163 & $0526-52312-445$ & 5.918 & 0.254 & 0.075 & II & 1995 I \\
\hline 84 & 075646 & 365917 & 119.19379425 & 36.98800659 & $0543-52017-002$ & 5.567 & 0.264 & 0.077 & Ia & $2001 \mathrm{ks}$ \\
\hline 85 & 083210 & 471728 & 128.04103088 & 47.29100800 & $0549-51981-379$ & 8.624 & 0.213 & 0.133 & Ia & $2001 \mathrm{kn}$ \\
\hline 86 & 103926 & 050524 & 159.86250305 & 5.09011221 & $0577-52367-591$ & 3.264 & 0.399 & 0.072 & Ia & 2008 iq \\
\hline 87 & 103929 & 051101 & 159.86888123 & 5.18365812 & $0578-52339-326$ & 3.154 & 0.433 & 0.067 & Ia & 2006 al \\
\hline 88 & 110823.953 & 032953.72 & 167.09875488 & 3.50014853 & $0581-52356-130$ & 27.924 & 0.409 & 0.024 & Ia & $2003 \mathrm{eh}$ \\
\hline 89 & 161713 & 482828 & 244.30583191 & 48.47438812 & 0622-52054-011 & 3.325 & 0.398 & 0.103 & Ia & $2001 \mathrm{kt}$ \\
\hline 90 & 204757 & -052425 & 311.98709106 & -5.40590429 & $0635-52145-489$ & 6.541 & 0.167 & 0.046 & Ia & $2007 \mathrm{hk}$ \\
\hline 91 & 001333 & -101309 & 3.38948441 & -10.21913052 & 0651-52141-034 & 6.692 & 0.193 & 0.109 & Ia & 2002 iu \\
\hline 92 & 003900.200 & -090053.20 & 9.75100803 & -9.01458073 & $0655-52162-368$ & 7.745 & 0.210 & 0.020 & Ia & 2006 ej \\
\hline 93 & 004113 & -090900 & 10.30378246 & -9.15011024 & $0655-52162-532$ & 5.059 & 0.331 & 0.053 & Ia & $2009 \mathrm{ly}$ \\
\hline 94 & 004459 & -085311 & 11.24682903 & -8.88971710 & $0656-52148-404$ & 11.059 & 0.187 & 0.019 & Ia & $2003 \mathrm{im}$ \\
\hline 95 & 020419.002 & -084407.24 & 31.07903671 & -8.73529243 & $0666-52149-638$ & 12.432 & 0.152 & 0.017 & Ia & 2006 ef \\
\hline 96 & 215721 & -075124 & 329.34158325 & -7.85690928 & $0716-52203-494$ & 5.819 & 0.282 & 0.056 & Ia & 2002 ep \\
\hline 97 & 222712 & -092944 & 336.80273438 & -9.49496269 & $0720-52206-003$ & 8.155 & 0.151 & 0.056 & Ia & $1995 \mathrm{~T}$ \\
\hline 98 & 225400 & 143924 & 343.50265503 & 14.65674591 & $0741-52261-425$ & 6.949 & 0.194 & 0.038 & Ia & $2002 \mathrm{fs}$ \\
\hline 99 & 230548 & 141956 & 346.45358276 & 14.33142471 & $0742-52263-590$ & 7.429 & 0.209 & 0.108 & Ia & $2012 \mathrm{ff}$ \\
\hline 100 & 231225 & 135449 & 348.10827637 & 13.91394138 & $0744-52251-228$ & 6.047 & 0.261 & 0.033 & Ia & $2007 \mathrm{ob}$ \\
\hline 101 & 231742 & 135724 & 349.42849731 & 13.95838356 & $0745-52258-297$ & 9.153 & 0.164 & 0.055 & Ia & 2009 jp \\
\hline 102 & 232748 & 142827 & 351.95312500 & 14.47530746 & $0746-52238-193$ & 14.404 & 0.162 & 0.041 & Ia & $2006 \mathrm{da}$ \\
\hline 103 & 233056.797 & 152925.95 & 352.74032593 & 15.48952103 & $0746-52238-564$ & 17.105 & 0.217 & 0.013 & II & 2011 ef \\
\hline 104 & 001911 & 150623 & 4.79587030 & 15.10631943 & $0752-52251-634$ & 5.880 & 0.205 & 0.014 & II & 1995 ah \\
\hline 105 & 090932.922 & 501654.84 & 137.38726807 & 50.28185272 & $0766-52247-316$ & 9.831 & 0.151 & 0.016 & Ia & $2001 \mathrm{G}$ \\
\hline 106 & 093836.273 & 521742.06 & 144.65115356 & 52.29494476 & $0768-52281-131$ & 8.215 & 0.221 & 0.050 & Ia & $2003 \mathrm{kz}$ \\
\hline 107 & 144443 & 585542 & 221.18432617 & 58.92900467 & $0790-52441-620$ & 6.428 & 0.199 & 0.044 & II & 1999 ay \\
\hline 108 & 113240.195 & 525701.06 & 173.16769409 & 52.95038605 & $0879-52365-580$ & 7.024 & 0.262 & 0.026 & Ia & 2002 bn \\
\hline 109 & 111609 & 552930 & 169.03520203 & 55.49029541 & $0909-52379-214$ & 5.677 & 0.258 & 0.057 & Ia & 1992 B \\
\hline 110 & 101857.961 & 462714.72 & 154.74162292 & 46.45409393 & 0944-52614-505 & 7.526 & 0.168 & 0.029 & Ic & $2003 \mathrm{ds}$ \\
\hline 111 & 105741 & 573648 & 164.42208862 & 57.61346436 & $0949-52427-109$ & 6.375 & 0.193 & 0.080 & Ia & $2010 \mathrm{bg}$ \\
\hline 112 & 104438 & 574840 & 161.15913391 & 57.81105804 & $0949-52427-310$ & 3.274 & 0.428 & 0.118 & Ia & $2010 \mathrm{bb}$ \\
\hline 113 & 203843 & -002828 & 309.68264771 & -0.47638249 & 0981-52435-205 & 2.970 & 0.200 & 0.147 & Ia & $2006 \mathrm{ex}$ \\
\hline 114 & 203648 & 000554 & 309.20251465 & 0.09849742 & $0981-52435-334$ & 2.383 & .511 & 0.146 & Ia & $2007 \mathrm{js}$ \\
\hline 115 & 204711 & -011526 & 311.79736328 & -1.25806630 & 0982-52466-198 & 6.175 & 0.221 & 0.057 & II & $2007 \mathrm{nw}$ \\
\hline 116 & 205452 & -001141 & 313.71878052 & -0.19580916 & $0983-52443-114$ & 3.525 & 0.399 & 0.174 & Ia & $2006 \mathrm{ni}$ \\
\hline 117 & 205209 & -003040 & 313.03787231 & -0.51093042 & $0983-52443-183$ & 4.430 & 0.281 & 0.070 & Ia & $2006 \mathrm{fe}$ \\
\hline 118 & 205036 & -002114 & 312.65274048 & -0.35423487 & $0983-52443-271$ & 11.305 & 0.195 & 0.258 & Ia & $2005 \mathrm{gh}$ \\
\hline 119 & 210308 & -010145 & 315.78494263 & -1.03117168 & 0984-52442-011 & 11.304 & 0.195 & 0.139 & Ia & $2007 \mathrm{hz}$ \\
\hline 120 & 210959 & 002431 & 317.49566650 & 0.40859288 & $0985-52431-587$ & 5.510 & 0.266 & 0.099 & Ia & $2006 \mathrm{fS}$ \\
\hline 121 & 211532 & -002119 & 318.88247681 & -0.35459545 & $0986-52443-086$ & 4.710 & 0.332 & 0.197 & Ia & $2005 \mathrm{kn}$ \\
\hline 122 & 212837 & 011341 & 322.15667725 & 1.23017097 & $0988-52520-511$ & 6.801 & 0.189 & 0.049 & Ia & 2006 eq \\
\hline 123 & 213530 & -005849 & 323.87850952 & -0.97963822 & $0989-52468-122$ & 3.379 & 0.390 & 0.167 & Ia & $2006 \mathrm{fa}$ \\
\hline 124 & 114811.320 & 545930.19 & 177.04718018 & 54.99180603 & $1016-52759-228$ & 11.156 & 0.151 & 0.008 & II & 2006 iv \\
\hline 125 & 131446.547 & 540514.69 & 198.69383240 & 54.08740234 & $1040-52722-488$ & 9.380 & 0.204 & 0.033 & Ia & 2012 ge \\
\hline 126 & 141346 & 521317 & 213.44218445 & 52.22148895 & $1045-52725-378$ & 5.702 & 0.223 & 0.077 & Ia & $2012 \mathrm{dm}$ \\
\hline 127 & 161743 & 345755 & 244.43147278 & 34.96490860 & $1057-52522-315$ & 6.634 & 0.239 & 0.027 & Ia & $2006 \mathrm{dw}$ \\
\hline 128 & 074726 & 265532 & 116.86002350 & 26.92566872 & $1059-52618-003$ & 5.498 & 0.232 & 0.015 & Ic & $2005 \mathrm{kf}$ \\
\hline 129 & 161921 & 410523 & 244.84024048 & 41.08987808 & $1171-52753-185$ & 8.646 & 0.192 & 0.037 & Ia & $2003 \mathrm{~lx}$ \\
\hline 130 & 163214 & 383920 & 248.05726624 & 38.65555954 & $1173-52790-537$ & 8.355 & 0.150 & 0.039 & II & $2012 \mathrm{ct}$ \\
\hline 131 & 094628.555 & 454509.12 & 146.61911011 & 45.75260162 & $1202-52672-604$ & 10.014 & 0.167 & 0.015 & Ia & $2003 \mathrm{jz}$ \\
\hline 132 & 114154 & 102546 & 175.47506714 & 10.43022156 & $1225-52760-463$ & 6.341 & 0.279 & 0.151 & Ia & 2009 be \\
\hline 133 & 123443.547 & 090017.02 & 188.68148804 & 9.00471687 & $1233-52734-250$ & 8.235 & 0.224 & 0.043 & Ia & $1993 \mathrm{I}$ \\
\hline 134 & 094530 & 063225 & 146.37681580 & 6.53983545 & $1234-52724-114$ & 4.816 & 0.315 & 0.087 & Ia & $2009 \mathrm{cj}$ \\
\hline 135 & 035157 & -002347 & 57.98822021 & -0.39662281 & $1242-52901-110$ & 3.221 & 0.416 & 0.165 & II & 2006 qf \\
\hline 136 & 091236.164 & 345118.84 & 138.15080261 & 34.85499954 & $1273-52993-477$ & 5.963 & 0.171 & 0.061 & Ia & $2010 \mathrm{au}$ \\
\hline 137 & 142556 & 462656 & 216.48419189 & 46.44973755 & $1287-52728-614$ & 7.189 & 0.184 & 0.033 & II & $2007 \mathrm{fe}$ \\
\hline 138 & 100544.539 & 101636.23 & 151.43548584 & 10.27677631 & $1308-53053-176$ & 11.614 & 0.171 & 0.024 & Ia & 2004 ap \\
\hline 139 & 112851 & 570804 & 172.21507263 & 57.13423920 & $1310-53033-432$ & 4.910 & 0.210 & 0.077 & Ia & $1999 \mathrm{ce}$ \\
\hline 140 & 114748.141 & 560106.25 & 176.95053101 & 56.01832962 & $1311-52765-055$ & 7.947 & 0.209 & 0.054 & Ia & $2000 \mathrm{~K}$ \\
\hline 141 & 134139.609 & 554014.69 & 205.41357422 & 55.66810226 & $1322-52791-198$ & 11.339 & 0.730 & 0.025 & Ic & 2001 ai \\
\hline 142 & 162148 & 370341 & 245.45220947 & 37.06080627 & $1337-52767-086$ & 6.969 & 0.193 & 0.029 & $\mathrm{Ib}$ & $2008 \mathrm{fn}$ \\
\hline 143 & 163320 & 344820 & 248.33615112 & 34.80719757 & $1339-52767-430$ & 7.549 & 0.154 & 0.035 & II & $1988 \mathrm{Q}$ \\
\hline 144 & 165222.656 & 304240.00 & 253.09448242 & 30.71103859 & $1343-52790-535$ & 10.091 & 0.165 & 0.036 & Ia & $2002 \mathrm{di}$ \\
\hline 45 & 115538 & & 178.90960693 & 44.38381958 & $1368-53084-097$ & 8.224 & 0.154 & 0.023 & II & $2006 \mathrm{db}$ \\
\hline 146 & 115017 & 435745 & 177.57095337 & 43.96262360 & $1368-53084-237$ & 2.745 & 0.484 & 0.071 & Ia & $2010 \mathrm{kn}$ \\
\hline
\end{tabular}


TABLE 4

-CONTINUED

\begin{tabular}{|c|c|c|c|c|c|c|c|c|c|c|}
\hline No. & RA (ASC) & DEC (ASC) & RA (SDSS) & DEC (SDSS) & PID-MJD-FID & $\begin{array}{c}\text { Petrion } \\
\text { radius }\end{array}$ & $\begin{array}{c}\text { light } \\
\text { fraction }\end{array}$ & redshift & Type & name of SN \\
\hline 147 & 121937 & 460157 & 184.90582275 & 46.03244019 & $1371-52821-325$ & 4.400 & 0.252 & 0.057 & II & $2010 \mathrm{bd}$ \\
\hline 148 & 152045 & 364842 & 230.18786621 & 36.81179428 & $1400-53470-234$ & 5.800 & 0.293 & 0.103 & Ia & $2005 \mathrm{bm}$ \\
\hline 149 & 165311 & 235754 & 253.29701233 & 23.96512794 & $1424-52912-509$ & 3.208 & 0.433 & 0.047 & Ia & $2009 \mathrm{fx}$ \\
\hline 150 & 115345 & 482521 & 178.43844604 & 48.42248535 & $1446-53080-454$ & 8.335 & 0.159 & 0.052 & Ia & $2008 \mathrm{ac}$ \\
\hline 151 & 120957 & 470543 & 182.48628235 & 47.09600067 & $1449-53116-070$ & 7.033 & 0.228 & 0.031 & Ia & $2006 \mathrm{ct}$ \\
\hline 152 & 122435 & 471416 & 186.14967346 & 47.23744965 & $1451-53117-073$ & 5.654 & 0.265 & 0.163 & Ia & 2009 co \\
\hline 153 & 131523 & 462509 & 198.84954834 & 46.42040253 & $1461-53062-166$ & 8.732 & 0.162 & 0.056 & II & $2009 \mathrm{ct}$ \\
\hline 154 & 160205 & 294334 & 240.52136230 & 29.72726059 & $1578-53496-421$ & 7.051 & 0.175 & 0.014 & II & $2007 \mathrm{fz}$ \\
\hline 155 & 154024 & 325157 & 235.10314941 & 32.86589813 & $1581-53149-470$ & 6.074 & 0.267 & 0.053 & Ia & $2004 \mathrm{cp}$ \\
\hline 156 & 102250 & 114211 & 155.70881653 & 11.70301819 & $1598-53033-380$ & 6.743 & 0.224 & 0.101 & Ia & $2004 \mathrm{cj}$ \\
\hline 157 & 121621 & 123138 & 184.08850098 & 12.52778912 & $1612-53149-015$ & 15.051 & 0.174 & 0.064 & Ia & $1990 \mathrm{~J}$ \\
\hline 158 & 121952 & 074349 & 184.96885681 & 7.73122072 & $1625-53140-499$ & 8.354 & 0.164 & 0.012 & II & 1997 bo \\
\hline 159 & 122450 & 082557 & 186.20838928 & 8.43370342 & $1626-53472-419$ & 7.143 & 0.174 & 0.090 & Ic & $2009 \mathrm{bh}$ \\
\hline 160 & 142355 & 351105 & 215.98291016 & 35.18551636 & $1644-53144-167$ & 6.184 & 0.179 & 0.055 & Ia & 2009 av \\
\hline 161 & 145000 & 445505 & 222.49903870 & 44.91716766 & $1675-53466-081$ & 5.545 & 0.235 & 0.080 & Ia & $2009 \mathrm{fb}$ \\
\hline 162 & 152748 & 413534 & 231.95225525 & 41.59374619 & $1679-53149-427$ & 5.938 & 0.168 & 0.081 & Ia & $2009 \mathrm{fc}$ \\
\hline 163 & 160209 & 364308 & 240.53713989 & 36.72050858 & $1682-53173-453$ & 4.816 & 0.237 & 0.094 & Ia & $2001 \mathrm{bp}$ \\
\hline 164 & 170925.078 & 221250.45 & 257.35443115 & 22.21404839 & $1688-53462-009$ & 2.413 & 0.614 & 0.048 & Ia & 2010 ed \\
\hline 165 & 170007 & 230756 & 255.02867126 & 23.13151169 & $1688-53462-310$ & 7.485 & 0.220 & 0.056 & Ia & $2008 \mathrm{eq}$ \\
\hline 166 & 133238 & 114833 & 203.16078186 & 11.80923462 & $1700-53502-359$ & 4.738 & 0.265 & 0.150 & Ia & 2005 ca \\
\hline 167 & 145643 & 091942 & 224.18005371 & 9.32679081 & $1715-54212-190$ & 7.902 & 0.207 & 0.079 & Ia & $2005 \mathrm{ag}$ \\
\hline 168 & 151836 & 095117 & 229.65385437 & 9.85440731 & $1720-53854-255$ & 5.534 & 0.228 & 0.032 & II & $2007 \mathrm{dp}$ \\
\hline 169 & 161412 & 060904 & 243.54829407 & 6.15103197 & $1731-53884-181$ & 8.926 & 0.190 & 0.039 & Ia & $2000 \mathrm{df}$ \\
\hline 170 & 082933 & 085205 & 127.38945770 & 8.86817551 & $1758-53084-523$ & 4.566 & 0.329 & 0.112 & Ia & $2004 \mathrm{cl}$ \\
\hline 171 & 120940 & 161212 & 182.41508484 & 16.20340538 & $1765-53466-368$ & 4.339 & 0.296 & 0.076 & Ia & $2013 \mathrm{Y}$ \\
\hline 172 & 090456.859 & 595558.69 & 136.23130798 & 59.93263245 & $1785-54439-632$ & 2.454 & 0.746 & 0.005 & Ic & $1995 \mathrm{~F}$ \\
\hline 173 & 152038 & 073932 & 230.15852356 & 7.65969324 & $1818-54539-508$ & 4.746 & 0.336 & 0.045 & Ia & $2009 \mathrm{eh}$ \\
\hline 174 & 153452 & 070053 & 233.71890259 & 7.01332378 & $1820-54208-481$ & 6.466 & 0.273 & 0.074 & II & 2007 ed \\
\hline 175 & 155113.266 & 254207.41 & 237.80531311 & 25.70191193 & $1850-53786-555$ & 12.155 & 0.150 & 0.021 & Ia & $2009 \mathrm{dc}$ \\
\hline 176 & 162034 & 211208 & 245.14274597 & 21.20253754 & 1853-53566-076 & 7.988 & 0.219 & 0.032 & Ia & 2011 bk \\
\hline 177 & 074837 & 521322 & 117.15312958 & 52.22221375 & $1869-53327-355$ & 9.383 & 0.197 & 0.064 & Ia & 1997 ea \\
\hline 178 & 092822 & 272640 & 142.09411621 & 27.44466400 & $1940-53383-478$ & 3.800 & 0.372 & 0.032 & Ia & 2003 ae \\
\hline 179 & 095847.523 & 344709.59 & 149.69892883 & 34.78640747 & $1948-53388-558$ & 2.532 & 0.807 & 0.017 & Ia & 2011 hd \\
\hline 180 & 103610 & 343233 & 159.04226685 & 34.54280472 & $1982-53436-445$ & 8.805 & 0.176 & 0.051 & Ia & 2007 do \\
\hline 181 & 124538.609 & 350501.78 & 191.41104126 & 35.08375549 & $1987-53765-329$ & 14.577 & 0.173 & 0.032 & Ia & $2006 \mathrm{~S}$ \\
\hline 182 & 130502 & 284424 & 196.26057434 & 28.73899651 & 2011-53499-020 & 7.033 & 0.238 & 0.027 & Ia & $2006 \mathrm{cg}$ \\
\hline 183 & 111229 & 312305 & 168.12567139 & 31.38496208 & $2092-53460-516$ & 6.309 & 0.176 & 0.027 & $\mathrm{Ib}$ & $2011 \mathrm{bp}$ \\
\hline 184 & 120323 & 351933 & 180.84963989 & 35.32583237 & $2103-53467-081$ & 4.156 & 0.321 & 0.028 & II & $2005 \mathrm{bn}$ \\
\hline 185 & 131651.141 & 313452.62 & 199.21447754 & 31.57986069 & $2104-53852-427$ & 13.090 & 0.191 & 0.029 & Ia & $2004 \mathrm{E}$ \\
\hline 186 & 135806.094 & 282522.12 & 209.52339172 & 28.42267036 & $2118-53820-535$ & 8.346 & 0.454 & 0.026 & II & $2000 \mathrm{ck}$ \\
\hline 187 & 144823 & 214751 & 222.09696960 & 21.79764938 & $2144-53770-215$ & 5.419 & 0.277 & 0.155 & Ia & 2006 ae \\
\hline 188 & 150030.203 & 235545.91 & 225.12585449 & 23.92932510 & $2152-53874-280$ & 10.522 & 0.181 & 0.047 & Ia & 2012 af \\
\hline 189 & 160527.281 & 174951.84 & 241.36349487 & 17.83099174 & 2199-53556-118 & 9.340 & 0.180 & 0.034 & Ia & $2000 \mathrm{cp}$ \\
\hline 190 & 111821 & 281243 & 169.59053040 & 28.20896530 & $2215-53793-088$ & 9.862 & 0.173 & 0.068 & Ia & $2006 \mathrm{bm}$ \\
\hline 191 & 111158 & 294205 & 167.99351501 & 29.69836235 & $2215-53793-350$ & 3.690 & 0.376 & 0.055 & Ia & 2012 ax \\
\hline 192 & 124937 & 281946 & 192.40368652 & 28.32912064 & $2238-54205-614$ & 4.022 & 0.365 & 0.055 & Ia & $2008 \mathrm{ad}$ \\
\hline 193 & 125541.328 & 271502.59 & 193.92207336 & 27.25076485 & $2240-53823-200$ & 8.830 & 0.199 & 0.023 & Ia & $2001 \mathrm{cg}$ \\
\hline 194 & 125924 & 282051 & 194.85052490 & 28.34720230 & $2240-53823-566$ & 5.998 & 0.247 & 0.067 & Ia & $2006 \mathrm{cj}$ \\
\hline 195 & 125925 & 275948 & 194.85437012 & 27.99674225 & $2241-54169-481$ & 7.133 & 0.207 & 0.018 & Ia & 2010 ai \\
\hline 196 & 093606 & 242413 & 144.02465820 & 24.40494728 & 2294-53733-006 & 7.692 & 0.169 & 0.070 & Ia & $2005 \mathrm{kw}$ \\
\hline 197 & 100006 & 281652 & 150.02825928 & 28.28054428 & $2345-53757-472$ & 18.021 & 0.157 & 0.089 & Ia & $2003 \mathrm{bh}$ \\
\hline 198 & 103357 & 202025 & 158.48828125 & 20.34046555 & $2376-53770-183$ & 4.666 & 0.336 & 0.087 & Ia & 2006 af \\
\hline 199 & 115004 & 211647 & 177.51690674 & 21.27984238 & $2511-53882-115$ & 6.521 & 0.274 & 0.025 & Ia & $2013 \mathrm{~N}$ \\
\hline 200 & 114328 & 214030 & 175.86759949 & 21.67408752 & $2511-53882-247$ & 8.222 & 0.198 & 0.068 & Ia & $2004 \mathrm{Y}$ \\
\hline 201 & 115614 & 252111 & 179.06001282 & 25.35412598 & $2514-53882-549$ & 8.475 & 0.176 & 0.032 & Ia & 2011 bg \\
\hline 202 & 162345.031 & 094716.92 & 245.93763733 & 9.78810501 & $2532-54589-360$ & 8.316 & 0.216 & 0.034 & Ia & $2012 \mathrm{ds}$ \\
\hline 203 & 100633.289 & 142601.20 & 151.63864136 & 14.43352890 & $2586-54169-158$ & 9.686 & 0.180 & 0.029 & II & $2013 \mathrm{~W}$ \\
\hline 204 & 100919 & 145932 & 152.33206177 & 14.99094009 & $2586-54169-567$ & 9.483 & 0.154 & 0.030 & Ia & 2007 ux \\
\hline 205 & 102311 & 175906 & 155.79826355 & 17.98383331 & $2589-54174-541$ & 8.568 & 0.211 & 0.027 & Ia & 1999 at \\
\hline 206 & 123936 & 163516 & 189.89941406 & 16.58782768 & 2599-54234-006 & 3.961 & 0.353 & 0.025 & Ia & $2012 \mathrm{G}$ \\
\hline 207 & 130950 & 204442 & 197.46063232 & 20.74431992 & $2617-54502-531$ & 5.778 & 0.210 & 0.095 & Ia & $2007 \mathrm{eg}$ \\
\hline 208 & 145055 & 171312 & 222.73104858 & 17.21818733 & $2777-54554-132$ & 7.295 & 0.159 & 0.040 & II & $2004 \mathrm{~V}$ \\
\hline 209 & 144747 & 164953 & 221.94726562 & 16.83006668 & $2777-54554-289$ & 9.755 & 0.166 & 0.045 & Ia & 2007 ee \\
\hline 210 & 144755.609 & 190326.09 & 221.98185730 & 19.05737495 & $2777-54554-331$ & 6.431 & 0.252 & 0.042 & Ia & $2010 \mathrm{cs}$ \\
\hline 211 & 145415 & 185752 & 223.56640625 & 18.96429634 & $2778-54539-336$ & 5.738 & 0.316 & 0.058 & Ia & 2009 eg \\
\hline 212 & 140051 & 225728 & 210.21182251 & 22.95813370 & $2784-54529-473$ & 7.191 & 0.165 & 0.083 & Ia & $2007 \mathrm{dv}$ \\
\hline 213 & 122248 & 053624 & 185.69837952 & 5.60674953 & $2880-54509-413$ & 7.857 & 0.221 & 0.017 & II & $2012 \mathrm{ab}$ \\
\hline
\end{tabular}

